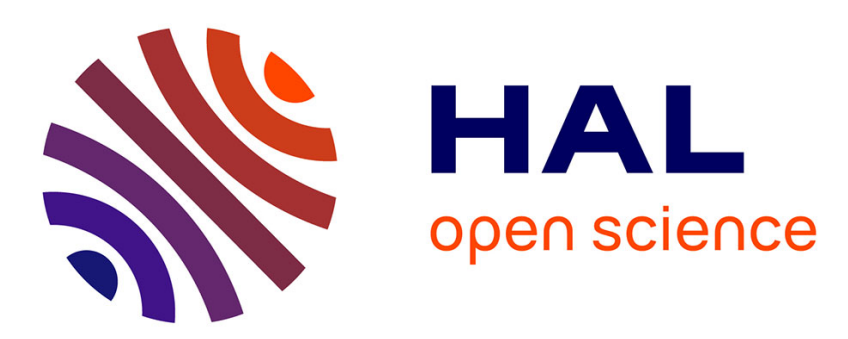

\title{
The structure of polyelectrolyte complex coacervates and multilayers
}

Andrey V Subbotin, Alexander N Semenov

\section{To cite this version:}

Andrey V Subbotin, Alexander N Semenov. The structure of polyelectrolyte complex coacervates and multilayers. Macromolecules, 2021, 54 (3), pp.1314-1328. 10.1021/acs.macromol.0c02470 . hal03255156

\section{HAL Id: hal-03255156 \\ https://hal.science/hal-03255156}

Submitted on 9 Jun 2021

HAL is a multi-disciplinary open access archive for the deposit and dissemination of scientific research documents, whether they are published or not. The documents may come from teaching and research institutions in France or abroad, or from public or private research centers.
L'archive ouverte pluridisciplinaire HAL, est destinée au dépôt et à la diffusion de documents scientifiques de niveau recherche, publiés ou non, émanant des établissements d'enseignement et de recherche français ou étrangers, des laboratoires publics ou privés. 


\section{The structure of polyelectrolyte complex coacervates and multilayers}

Andrey V. Subbotin ${ }^{*}, \mathrm{a}, \mathrm{b}$ and Alexander N. Semenov ${ }^{\mathrm{c}}$

${ }^{a}$ A.V. Topchiev Institute of Petrochemical Synthesis, Russian Academy of Sciences, Leninskii prosp. 29, Moscow, 119991, Russia.

${ }^{b}$ A.N. Frumkin Institute of Physical Chemistry and Electrochemistry, Russian Academy of Sciences, Leninskii prosp. 31, Moscow, 119071, Russia.

${ }^{c}$ Institut Charles Sadron, CNRS - UPR 22, Université de Strasbourg, 23 rue du Loess, 67034 Strasbourg Cedex 2, France.

*Corresponding author: Andrey Subbotin

E-mail: subbotin@ips.ac.ru 
Polycation (PC)+Polyanion (PA)+salt+solvent

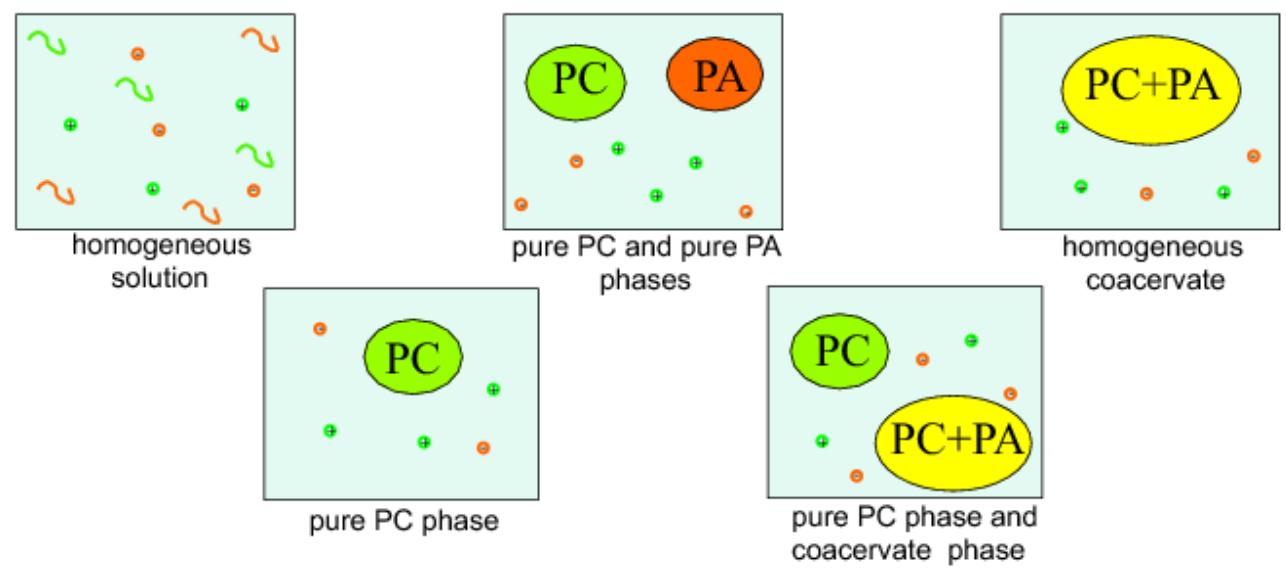

\begin{abstract}
:
Equilibrium structure of the polyelectrolyte (PE) complex coacervates and homogeneous multilayers formed by polycations and polyanions with incompatible backbones in a poor solvent is studied using a mean-field approach. The state diagrams involving symmetric and asymmetric homogeneous phases are obtained in terms of interaction parameters, ionic strength and composition. It is found that interfacial tension between two coexisting uniform PE phases vanishes at a certain line, where both phases remain stable and (unlike the classical critical behavior) highly distinct in composition and charge. It is also shown that a microdomain structure of the coacervate phase gets thermodynamically stable at low enough salinity. Lamellar structures arising in symmetric coacervates are investigated in all segregation regimes (weak, strong and intermediate). The mechanism of an exponential growth of uniform multilayer thickness upon layer-by-layer deposition is elucidated. It is predicted that the exponential growth rate increases with the solution ionic strength in agreement with experimental data.
\end{abstract}




\section{Introduction}

The ability of polyelectrolytes (PEs) to form complex coacervate phases and multilayers as well as their decisive role in the functioning of living systems make these objects interesting for experimental study and theoretical analysis and it also allows them to be used for a variety of applications. ${ }^{1-4}$ In the last decades, significant progress has been made in understanding the behavior of PE systems. ${ }^{5}$ Meanwhile, many questions related to the self-assembly of charged polyelectrolyte chains into various spatial structures, including the formation of polyelectrolyte multilayers, remain open. Progress in this field is important for the development of new functional materials.

Polymer chains get charged as a result of electrostatic dissociation of ionic groups. It is well-known that in dilute solutions such PE chains adopt an extended conformation. ${ }^{6,7}$ In good solvents at low ionic strength (low salinity) conformations of PE chains can be described as a sequence of blobs, ${ }^{6-8}$ while in poor solvents the chains become partially collapsed forming a sequence of globules. ${ }^{9-12}$ An increase of salt concentration screens the electrostatic interactions and leads to bending fluctuations of PE chains, which can be characterized by the electrostatic persistence length. ${ }^{13}$

The tendency of counterions to occupy the maximum volume brings about an increase of solubility of PE chains rendering them soluble even in the case of insoluble backbones. It gives a number of interesting effects, including formation of microphase structures. ${ }^{14}$ Stabilization of these structures in the solutions of polyions of the same type (either cation or anion) is related to interplay between immiscibility of the PE chain backbone with the solvent on the one hand and their tendency to mix due to translational entropy of counterions on the other hand. An analysis of microdomain structures in weakly charged polyelectrolyte solutions in the weak segregation limit reveal that BCC, triangular and lamellar microdomain structures can be stable depending 
on PE concentration, ion strength and other solution parameters. ${ }^{11,15-18}$ An association between ions and dielectric mismatch between polymer and solvent result in both the macrophase separation and formation of new microdomain phases characterized by ionomer-type behavior in the polymer-rich region. ${ }^{19}$ Microphases also arise in the blends of incompatible polymers, if one of the polymers carries a charge..$^{20,21}$ Phase equilibria between homogeneous phase and microphases of different symmetry had been considered by Dobrynin and Erukhimovich ${ }^{20}$ using weak segregation theory whereas the lamellar phase had been analyzed by Nyrkova, Khokhlov and $\mathrm{Doi}^{21}$ both in strong and intermediate regimes by direct minimization of the free energy. Recently, microphase separation in salt free blends of oppositely charged polyelectrolytes with immiscible backbones had been studied both using the Leibler's approach and the BrazovskiiFredrickson-Helfand approximation, ${ }^{22}$ and the strong segregation theory which was verified by dissipative particle dynamic (DPD) simulations. ${ }^{23}$ It was also shown that block-copolymers containing polyelectrolyte blocks tend to form charged micelles. ${ }^{24,25}$

Complex coacervates (liquid phases where oppositely charged polymer segments are rather densely mixed) arise in PE solutions containing both polycations and polyanions or polyampholytes. ${ }^{26-29}$ Early approaches to study the coacervates were based on the Debye-Hückel theory and did not take into account the polymer specific effects. ${ }^{30,31}$ Further studies using theoretical tools like the random phase approximation (RPA), ${ }^{32-35}$ scaling methods ${ }^{36,37}$ and fieldtheory approaches ${ }^{38,39}$ made it possible to better analyze the structure of complex coacervates formed by weakly charged polyelectrolytes and the conditions of their emergence. Homogeneous coacervate phases in composition-asymmetric solutions of polycations and polyanions had been analyzed ${ }^{40}$ using a simple liquid-state theory based on the mean spherical approximation. The effect of incompatibility between oppositely charged polyelectrolytes on microphases formation had been studied by Castelnovo and Joanny ${ }^{41}$ in the weak segregation regime with a theory based on the one-loop approximation going beyond the standard RPA. The impact of solvent quality 
and salt concentration for the state diagram of a symmetric coacervate had been considered by Rumyantsev, Zhulina and Borisov. ${ }^{42}$ In addition, the effects of salinity and the interaction parameters on the formation of macroscopic uniform and lamellar phases in blended solutions of polycations and polyanions have been recently examined using the RPA and scaling approaches 43

PE multilayers (PEMs), which can also be regarded as complex coacervates, remain theoretically less studied, despite a large amount of experimental data on these systems. ${ }^{44-52}$ Depending on the formation conditions, as well as the deposition time and the number of deposition steps, the thickness of the multilayers can grow exponentially or linearly. In a number of cases, a transition from exponential growth to linear was observed in the course of deposition. ${ }^{45-50}$ To date, the most explored simple analogs of PEMs are adsorbed PE monolayers formed by flexible PE chains on an oppositely charged solid surface. ${ }^{53-58}$ An important effect here is the ability of the adsorbed layer to overcharge the surface (reversing its total charge). ${ }^{54,55}$ The influence of various parameters on the degree of charge reversal was studied recently in ref. 58. Noteworthily, the effect of surface charge inversion by the adsorbed polyions was considered as a driving force for the multilayer growth. ${ }^{59,60}$ However, it was also demonstrated that strong short-range non-electrostatic attraction between two types of deposited polymer chains may be essential for the formation of multilayers. ${ }^{61}$

In the present paper we consider complex coacervates formed by incompatible polycations and polyanions in poor solvent. We study various phase equilibria in these systems including symmetric and asymmetric coacervate phases with both uniform and lamellar structure. The dependence of the lamellar and the total multilayer thickness on the number of deposition steps and other parameters is considered as well. 


\section{Model and free energy}

Let us consider dilute polyelectrolyte solution containing polycations (PC), polyanions (PA) and small monovalent ions with absolute charge $e$. Each charged monomer group of polyion also carries absolute charge $e$. We assume that PC and PA are flexible chains of equal polymerization index $N \gg 1$ and the same statistical segment length $a_{s}$. The length $a_{s}$ is defined by the Kuhn segment length $l_{K}$ and the length $l_{1}\left(l_{K}>l_{1}\right)$ of the repeat monomer unit: the chain coil size $R_{\text {coil }} \simeq a_{s} \sqrt{N}=\sqrt{l_{K} L}$ where the contour chain length $L=l_{1} N$, hence $a_{s}=\sqrt{l_{K} l_{1}}$. The fraction of charged groups on the polyions is $f$, with $f \ll 1$ and $N f \gg 1$, i.e. the polyion segments are weakly charged, but the chains as a whole are charged strongly.

It is well known that solutions of polyions of opposite sign tend to form coacervate phases. ${ }^{27}$ Below we first consider a single coacervate phase of volume $V$ in which concentration of PC segments is $n_{C}$ and PA segments is $n_{A}$, so that $\int_{V} n_{C} d V=\mathcal{N}_{C}, \int_{V} n_{A} d V=\mathcal{N}_{A}$, where $\mathcal{N}_{C} / N \gg 1$ and $\mathcal{N}_{A} / N \gg 1$ are the numbers of PC and PA chains, respectively. Short-range (excluded-volume) interactions between the chain segments are described in terms of the virial expansion for the excess free energy density ${ }^{7}$

$$
F_{i n t} \simeq T\left(-\frac{B}{2}\left(n_{A}^{2}+n_{C}^{2}\right)+v n_{A} n_{C}+\frac{C}{3}\left(n_{A}+n_{C}\right)^{3}\right)
$$

Here $T=k_{B} T_{a b s}$ ( $k_{B}$ is Boltzmann constant, $T_{a b s}$ is the absolute temperature), $-B, v$ are the second virial coefficients and $C$ is the third one. For simplicity it is assumed that the virial coefficients $-B, C$ are the same both for PC and PA chains. The virial expansion is valid at low

polymer volume fraction, $\phi_{p} \equiv n b^{3} \ll 1$, where $n=n_{A}+n_{C}$ and $b \sim\left(l_{1} d^{2}\right)^{1 / 3}$ is the characteristic 
monomer size ( $d$ is the chain thickness). Typically $b \sim 5 \AA, C / b^{6} \sim 1$ and $B \sim b^{3}\left(\frac{\Theta}{T}-1\right)$ ( $\Theta$ is $\Theta$-temperature). ${ }^{7}$ More precisely it is assumed that the total polymer concentration $n$ is low enough, so that $C n^{2} \ll 1$. The parameter $\tilde{v}=v+B>0$ reflects a non-electrostatic repulsion between $\mathrm{A}$ and $\mathrm{C}$ segments (corresponding to a positive Flory parameter $\chi_{A C} \sim \tilde{v} / b^{3}$, i.e., a tendency for their phase separation which is typical for neutral polymers): PC and PA chains are incompatible in the absence of charges if $\tilde{v}>0$. It is also natural to assume that backbones of the polyions are hydrophobic: $B>0(\Theta>T)$.

The free energy density of the coacervate in the framework of the mean-field approximation is presented as a sum

$$
\tilde{F}=F_{c o n f}+F_{i n t}+F_{i d}+F_{e l}
$$

The first term in eq (2) is the conformational energy of polyions,

$$
F_{\text {conf }} \simeq \frac{a^{2} T}{4}\left[\frac{\left(\nabla n_{A}\right)^{2}}{n_{A}}+\frac{\left(\nabla n_{C}\right)^{2}}{n_{C}}\right]
$$

where $a^{2}=a_{s}^{2} / 6$ and $\nabla$ is the gradient operator (nabla). The above equation is based on the assumption that the characteristic length-scale $D$ associated with the profiles $n_{A}$ and $n_{C}$ is much shorter than the coil size $R_{\text {coil }} \sim a \sqrt{N}$. Here we neglected the translational entropy of the polyions since the chains are very long: $R_{\text {coil }} \gg D$. The second term in eq 2 is short-range interaction energy, see eq 1 . The third term is the ideal-gas free energy of small ions,

$$
F_{i d} \simeq T\left[c_{+} \ln \left(c_{+} / \mathrm{e}\right)+c_{-} \ln \left(c_{-} / \mathrm{e}\right)\right]
$$


where $c_{+}$and $c_{-}$are concentrations of small monovalent cations and anions respectively (in particular due to added salt) and $\mathrm{e} \approx 2.718$. We assume that volume fraction of small ions is so low that the excluded volume interactions between them can be neglected; ditto for interactions of small ions with polymer segments (whose volume fraction is low as well). The last term is the electrostatic energy which we present in the form

$$
F_{e l} \simeq T\left[\rho \Phi-\frac{1}{2 \tilde{l}_{B}}(\nabla \Phi)^{2}\right]
$$

Here $\Phi=e \varphi / T$ and $\varphi$ is the electrostatic potential, $\rho=f\left(n_{C}-n_{A}\right)+c_{+}-c_{-}$is the volume charge density, $\tilde{l}_{B}=4 \pi l_{B}$ and $l_{B}=\frac{e^{2}}{\varepsilon T}$ is the Bjerrum length ( $\varepsilon$ is the dielectric constant of the medium). It is assumed that $\varepsilon$ does not significantly depend on the polymer concentration since the latter is low. Thus eq 2 is written in the form

$$
\begin{aligned}
\tilde{F} \simeq T & {\left[\frac{a^{2}}{4}\left(\frac{\left(\nabla n_{A}\right)^{2}}{n_{A}}+\frac{\left(\nabla n_{C}\right)^{2}}{n_{C}}\right)-\frac{B}{2}\left(n_{A}^{2}+n_{C}^{2}\right)+v n_{A} n_{C}+\frac{C}{3}\left(n_{A}+n_{C}\right)^{3}\right.} \\
& \left.+c_{+} \ln \left(c_{+} / \mathrm{e}\right)+c_{-} \ln \left(c_{-} / \mathrm{e}\right)+\left(f\left(n_{C}-n_{A}\right)+c_{+}-c_{-}\right) \Phi-\frac{1}{2 \tilde{l}_{B}}(\nabla \Phi)^{2}\right]
\end{aligned}
$$

Note that our approach is akin to, but is different from the mean-field theories developed in refs. 17, 21. The total free energy of the coacervate is $\mathcal{F}=\int_{V} \tilde{F} d V$. Maximization of the free energy $\mathcal{F}$ with respect to $\Phi$ results in the Poisson-Boltzmann equation

$$
\Delta \Phi=-\tilde{l}_{B}\left[f\left(n_{C}-n_{A}\right)+c_{+}-c_{-}\right]
$$

The dilute bulk phase (referred to as simply bulk phase hereafter) stays in thermodynamic equilibrium with the coacervate phase. The free energy density of the bulk phase containing 
small ions with concentration $c_{s}$ of each sign is $F^{(b)} \simeq 2 T c_{s} \ln \left(c_{s} / \mathrm{e}\right)$ (the fraction of the free polyions in the bulk is negligibly small and is omitted). Equilibrium between the small ions in the bulk and in the coacervate implies equality of their chemical potentials:

$$
\mu_{+}=\frac{\delta \mathcal{F}}{\delta c_{+}}=T \ln c_{s}, \quad \mu_{-}=\frac{\delta \mathcal{F}}{\delta c_{-}}=T \ln c_{s}
$$

Note that $\varphi$ is constant (which is set to 0 ) in the bulk phase. Therefrom we find

$$
c_{ \pm}=c_{s} \exp (\mp \Phi)
$$

and the Poisson-Boltzmann equation 7 is written in the form

$$
\Delta \Phi=\frac{1}{r_{D}^{2}} \sinh \Phi-\tilde{l}_{B} f\left(n_{C}-n_{A}\right)
$$

Here $r_{D}$ is the Debye length, $r_{D}=\left(2 \tilde{l}_{B} c_{s}\right)^{-1 / 2}$.

To analyze the coacervate structure it is convenient to use the semi-grand thermodynamic potential $\Delta \mathcal{F}=\mathcal{F}-\mu_{+} \mathcal{N}_{+}-\mu_{-} \mathcal{N}_{-}$, where $\mathcal{N}_{+}$and $\mathcal{N}_{-}$are the numbers of small ions in the coacervate. Thus $\Delta \mathcal{F}$ depends on $\mu_{+}$and $\mu_{-}$, but also on the concentration profiles of polymer segments:

$$
\begin{aligned}
\Delta \mathcal{F}=T \int_{V} d V[ & \frac{a^{2}}{4}\left(\frac{\left(\nabla n_{A}\right)^{2}}{n_{A}}+\frac{\left(\nabla n_{C}\right)^{2}}{n_{C}}\right)-\frac{B}{2}\left(n_{A}^{2}+n_{C}^{2}\right)+v n_{A} n_{C}+\frac{C}{3}\left(n_{A}+n_{C}\right)^{3} \\
& \left.-2 c_{s}(\cosh \Phi-1)+f\left(n_{C}-n_{A}\right) \Phi-\frac{1}{2 \tilde{l}_{B}}(\nabla \Phi)^{2}\right]
\end{aligned}
$$

\section{Homogeneous coacervate phases}


First we consider a homogeneous coacervate of volume $V$ with concentrations $n_{A}=\mathcal{N}_{A} / V=$ const,$n_{C}=\mathcal{N}_{C} / V=$ const . The free energy 10 in this case reads

$$
\Delta \mathcal{F}=T V\left[-\frac{B}{2}\left(n_{A}+n_{C}\right)^{2}+(B+v) n_{A} n_{C}+\frac{C}{3}\left(n_{A}+n_{C}\right)^{3}-2 c_{s}\left(\cosh \Phi_{0}-1\right)+f\left(n_{C}-n_{A}\right) \Phi_{0}\right]
$$

and $\Phi_{0}$ is defined by equation $\sinh \Phi_{0}=r_{D}^{2} \tilde{l}_{B} f\left(n_{C}-n_{A}\right)$ coming from eq $9 \mathrm{~b}$ where $\Delta \Phi=0$.

After introduction of a new variable $m=n_{C}-n_{A}(n \geq|m|)$ and using the Debye-Hückel approximation, $|\Phi| \ll 1$, eq 11 is written in a simple form

$$
\Delta \mathcal{F}=T V\left[-\frac{1}{4}(B-v) n^{2}+\frac{C}{3} n^{3}+\frac{1}{4}\left(B_{e s}-\tilde{v}\right) m^{2}\right]
$$

Here $B_{e s}=2 r_{D}^{2} \tilde{l}_{B} f^{2}=f^{2} / c_{s}$ is the electrostatic excluded volume. Next, let us introduce an asymmetry parameter $p=\frac{\mathcal{N}_{C}-\mathcal{N}_{A}}{\mathcal{N}_{C}+\mathcal{N}_{A}}=\frac{m}{n}$. The Debye-Hückel (DH) approximation for the electric potential requires that $f m \ll c_{s}$, i.e. $f p \phi_{p} \ll \phi_{s}$, where $\phi_{s}=c_{s} b^{3}$ is roughly the salt volume fraction. Another condition of validity of the DH-approximation reads: ${ }^{7} l_{B} / r_{D} \ll 1$ leading to $\phi_{s} \ll 1 /\left(8 \pi u^{3}\right) \sim 10^{-2}$ (here $u \equiv l_{B} / b$ is the standard Coulomb interaction parameter; with $l_{B} \sim 7 \AA, b \sim 5 \AA$ we get $\left.u \sim 1\right)$.

It is assumed that most of the total volume is occupied by the dilute bulk phase, where polymer concentration $n \rightarrow 0$ (in the case of long polymers, $N \rightarrow \infty$, considered here) and hence the osmotic pressure is zero. Below we identify the regions in the $(B, v)$ plane where one or several condensed phases are possible. By condensed phase we mean symmetric $(p=0)$ or asymmetric $(0<|p|<1)$ coacervate phases or pure phases of a single collapsed polyelectrolyte 
(A or $\mathrm{C},|p|=1$ ). For our multi-component system (PA $+\mathrm{PC}+$ small ions + solvent) the study of phase equilibrium can be based on the analysis of $F=\Delta \mathcal{F} /(T V n)$ considered as a function of $p$. Note that $F$ is proportional to the excess free energy per monomer (polymer segment) due to condensation of the added polyelectrolyte, and $p$ shows the gross composition depending on the total numbers of cation and anion chains in all the condensed phases. The phase equilibria in this system can be analyzed based on $F(p)$ using the common tangent construction in analogy with single-component systems (whose free energy density is analogous to $F$, and concentration or density - to $p$ ). In particular, $\frac{\partial^{2} F}{\partial p^{2}}<0$ signals an emergence of a phase separation region (that is, multiple coexisting condensed phases). Using eq 12 and the condition of zero osmotic pressure, $\Pi_{p}=-\frac{\partial \Delta \mathcal{F}}{\partial V}=0$, we get (for a single homogeneous condensed phase)

$$
F=-\frac{1}{3} C n^{2}=-\frac{3}{64 C} \Delta^{2}\left(A-p^{2}\right)^{2}, \quad n=\frac{3}{8 C} \Delta\left(A-p^{2}\right)
$$

where $\Delta=B_{e s}-\tilde{v}=B_{e s}-B-v, \quad A=(B-v) / \Delta$. The above equations are valid for $B-v-p^{2} \Delta>0$, that is $\Delta\left(A-p^{2}\right)>0$. Otherwise (if the latter condition is not respected) a homogeneous condensed phase cannot be formed.

Using eq 13 we identify 5 regions in the $(B, v)$ diagram (Fig.1a). In region I $\left(B<v, B<B_{e s} / 2\right)$ no condensed phase is possible (since $\Delta\left(A-p^{2}\right)$ is negative for any $p$ ), so the polyelectrolytes must be completely dissolved in the large-volume dilute bulk phase. This region consists of 2 subregions: Ia $(\Delta<0, A>1)$ and Ib $(\Delta>0, A<0)$. The next region II $B+v>B_{e s}, B>B_{e s} / 2$ (or, alternatively $\Delta<0, A<1$ ) corresponds to coexistence of 2 pure condensed polyelectrolyte phases: pure PC $(p=1)$ and pure PA $(p=-1)$, both of concentration 


$$
n=n_{I I}=\frac{3}{8 C}\left(2 B-B_{e s}\right)
$$

The coacervate condensed phases (symmetric or asymmetric) can be formed in the other 3 regions (III, IV, V). A single mixed condensed PE phase is stable in the region III $\left(v<B<B_{e s} / 2\right)$ if the global $p$ is such that $|p|<p^{*}$, where

$$
p^{*}=\frac{1}{3}(2-\sqrt{4-3 A}), \quad 0<p^{*}<1 / 3
$$

(Note that region III corresponds to $0<A<1, \Delta>0$ ). A more asymmetric coacervate with $|p|>p^{*}$ cannot stay in equilibrium with the dilute bulk (so any excess PC or PA must be dissolved in the bulk volume). Note that in this case the coacervate phase volume is defined by the amount of the minor PE segments, $\quad M=\min \left(\mathcal{N}_{A}, \mathcal{N}_{C}\right)$ : $V=(M / n)\left(2 /\left(1-p^{*}\right)=(6 M / n) /(1+\sqrt{4-3 A})\right.$ with $n=\Delta(3 A-2+\sqrt{4-3 A}) /(6 C)$.

Region IV $\left(2 B+v<1.5 B_{e s}, B>B_{e s} / 2\right)$ corresponds to $1<A<3, \Delta>0$. Depending on the global composition $p$, either a single condensed phase is formed in this region (if $|p|<p_{c}$ ) or otherwise (for $|p|>p_{c}$ ) it separates in 2 phases: a coacervate with $|p|=p_{c}$ and a pure PE phase (A or C) with $|p|=1$. Here

$$
p_{c}=\frac{1}{3}(\sqrt{6 A-2}-1)
$$

Finally, a single coacervate phase is always formed in region $\mathrm{V}\left(2 B+v>1.5 B_{e s}, B+v<B_{e s}\right)$ where $A>3, \Delta>0$. 


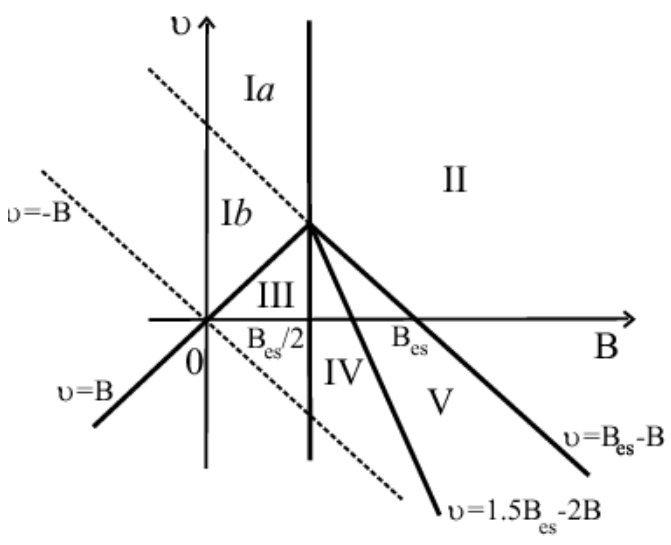

(a)

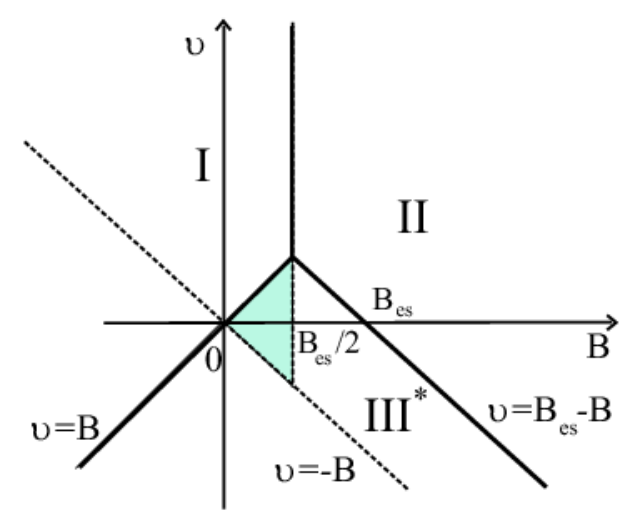

(b)

Figure 1. (a) Diagram of stable homogeneous phases in $(B, v)$ plane diagram for arbitrary composition of PE solution. (b) diagram of stable homogeneous phases in the symmetric case $(p=0)$. In the latter case all PE chains are condensed forming a common coacervate phase in the regions III, IV and V which are shown in part (b) as a unified region III". The shaded area in this region corresponds to soluble individual polyions $2 B<B_{e s}$ with incompatible backbones $(\tilde{v}>0)$.

Figure $1 \mathrm{~b}$ concerns the symmetric case, $p=0$. Here the coacervate phases are formed in the region III*; their density is defined in eq 25.

The thermodynamic and kinetic stability of all the coacervate phases considered above is ensured by high energy barriers which polyions have to overcome in order to enter the bulk solution (by kinetic stability here we mean that coacervate phases remain condensed and stable for a long time even in non-equilibrium conditions, in the absence of PEs in the surrounding bulk solution). The barrier height is defined by the chain chemical potential and is given by 


$$
\begin{aligned}
& U_{A}=-N \frac{\partial}{\partial n_{A}}\left(\frac{\Delta \mathcal{F}}{V}\right)=\frac{1}{8} T N n\left(B-v+p(3 p+4)\left(B_{e s}-\tilde{v}\right)\right) \\
& U_{C}=-N \frac{\partial}{\partial n_{C}}\left(\frac{\Delta \mathcal{F}}{V}\right)=\frac{1}{8} T N n\left(B-v+p(3 p-4)\left(B_{e s}-\tilde{v}\right)\right)
\end{aligned}
$$

For example when $p>0 \quad\left(\mathcal{N}_{C}>\mathcal{N}_{A}\right)$ both barriers are high if $B-v+p(3 p-4)\left(B_{e s}-\tilde{v}\right)>0$ which is equivalent to

$$
A+p(3 p-4)>0
$$

Note, that we consider the case $N \gg 1$ and the regimes III, IV, V where $B_{e s}>\tilde{v}, B>v$, i.e. $\Delta>0, A>0$. Therefore $U_{A}>U_{C}$ for $p>0$. The condition (18) means that either $p<p^{*}$ or $p>4 / 3-p^{*}$ if $A \leq 4 / 3$. In the opposite case, $A>4 / 3$ (i.e. $B-v>(4 / 3)\left(B_{e s}-\tilde{v}\right)$ ) the condition 18 is valid for any $p($ with $\Delta>0, A>0)$.

In the above analysis, we omitted the electrostatic fluctuation correction to the free energy. This energy is related to fluctuations of polymer charge density $\rho_{p}$. Using the relevant order parameter $m=n_{C}-n_{A}$ (the fluctuation $\delta \rho_{p}=f \delta m$ ) the free energy increment due to $\delta m$ is

$$
\delta \mathcal{F}=\frac{T V}{2} \int \frac{d^{3} \mathbf{q}}{(2 \pi)^{3}} \kappa(\mathbf{q})\left|m_{\mathbf{q}}\right|^{2}
$$

where $m_{\mathbf{q}}$ is the Fourier transform of $\delta m(\mathbf{r})$ with wave vector $\mathbf{q}$ and

$$
\kappa(\mathbf{q})=\frac{q^{2} a^{2}}{2 n}-\frac{\tilde{v}}{2}+\frac{f^{2} \tilde{l}_{B}}{r_{D}^{-2}+q^{2}}
$$


Here $q=|\mathbf{q}|$, the 1 st term comes from eq 3 , the 2 nd term corresponds to the second $\left(n_{A} n_{C}\right)$ term in eq 11, and the last term reflects electrostatic interactions screened by the salt. Employing the standard technique of parameter variation (of the Bjerrum length in the present case) based on the Landau's theorem on small increments, ${ }^{62}$ we get the correlation free energy (cp. ref. 37):

$$
\mathcal{F}_{\text {corr }} \simeq \frac{T V}{2} \int \frac{d^{3} \mathbf{q}}{(2 \pi)^{3}} \ln \left(\frac{\kappa(\mathbf{q})}{\kappa_{\text {ref }}(\mathbf{q})}\right)
$$

where $\kappa_{\text {ref }}(\mathbf{q})$ is $\kappa(\mathbf{q})$ for the reference system with switched off electrostatic interactions, $l_{B}=0$.

To simplify the problem let us assume $X \equiv\left(\frac{r_{D}^{2} n B_{e s}}{a^{2}}\right)^{1 / 2} \leq 1$ and set $\tilde{v}=0$ (the effect of $\tilde{v}$ is not important as long as $\left.B_{e s}>\tilde{v}\right)$. Then we get from eq 20

$$
\mathcal{F}_{\text {corr }} \simeq \frac{T V}{2} \int \frac{d^{3} \mathbf{Q}}{\left(2 \pi r_{D}\right)^{3}} \ln \left(1+\frac{X^{2}}{\mathbf{Q}^{2}} \frac{1}{1+\mathbf{Q}^{2}}\right)
$$

where $\mathbf{Q}=\mathbf{q} r_{D}$. It leads to

$$
\mathcal{F}_{\text {corr }} /(T V) \sim\left(X / r_{D}\right)^{3} \sim\left(n B_{e s}\right)^{3 / 2} / a_{s}^{3}=\left(f^{2} n / c_{s}\right)^{3 / 2} / a_{s}^{3}
$$

Comparing eq 22 with the main mean-field term $\Delta \mathcal{F} /(T V) \sim C n^{3}$ (cf. eq 13) and assuming that $B_{\text {es }} \leq C n$ we observe that $\mathcal{F}_{\text {corr }} \leq\left(C n^{2}\right)^{3 / 2} / a^{3}$ and it can be neglected if

$$
C^{1 / 2} / a_{s}^{3} \ll 1
$$


which is the standard condition of the mean-field theory validity in polymer physics. ${ }^{7}$ Lifting the assumption $B_{e s} \leq C n$ and using the inequalities $X \leq 1$ and $\mathcal{F}_{\text {corr }} \ll \Delta \mathcal{F}$ we arrive at an additional (sufficient) condition of negligible $\mathcal{F}_{\text {corr }}$ :

$$
f \ll a n^{3 / 2} C^{2 / 3} / l_{B}^{1 / 2}
$$

which is virtually equivalent to $f \ll \phi_{p}^{3 / 2} \tilde{C}^{2 / 3}$, where $\tilde{C}=C / b^{6}$. A simple analysis shows that if the basic condition (23) is valid, the condition (24) is always satisfied apart from a narrow zone near the boundary of region III with the dissolved phase I (cf. Fig. 1a) where the total concentration $n$ gets very small. Note also that eq. (24) can be written as $n \gg n^{*}$, where $n^{*} \sim\left(f / a_{s}\right)^{2 / 3} l_{B}^{1 / 3} C^{-4 / 9}$ is the typical coacervate concentration in a salt-free theta-solvent. ${ }^{35}$

\section{Stability analysis of the symmetric homogeneous phase}

In this part we consider stability of the symmetric coacervate phase $(p=0)$ with respect to microphase separation between PC and PA leading to a lamellar structure. The relevant phase is labeled III $^{*}$ in the diagram of PE solution with symmetric composition shown in Figure 1b. It is assumed that lamellar layers are oriented perpendicular to the $z$-axis. To begin with we consider the coacervate stability with respect to small fluctuations assuming that $|m| \ll \bar{n}$ and $|n-\bar{n}| \ll \bar{n}$ where

$$
\bar{n}=\frac{3}{8 C}(B-v)
$$


is concentration of the uniform symmetric phase. The free energy of the coacervate is presented in the form

$$
\Delta \mathcal{F}=T \int_{V}\left[-\frac{1}{4}(B-v) n^{2}+\frac{C}{3} n^{3}\right] d V+\Delta \mathcal{F}_{1}+\Delta \mathcal{F}_{2}
$$

where

$$
\begin{gathered}
\Delta \mathcal{F}_{1}=T \int_{V}\left[\frac{a^{2}}{4} \frac{n m_{z}^{\prime 2}}{n^{2}-m^{2}}-2 c_{s}(\cosh \Phi-1)+f m \Phi-\frac{1}{2 \tilde{l}_{B}} \Phi_{z}^{\prime 2}-\frac{1}{4} \tilde{v} m^{2}\right] d V \\
\Delta \mathcal{F}_{2}=T \frac{a^{2}}{4} \int_{V} \frac{n n_{z}^{\prime 2}-2 m n_{z}^{\prime} m_{z}^{\prime}}{n^{2}-m^{2}} d V
\end{gathered}
$$

and $n_{z}^{\prime}=\frac{d n}{d z}, m_{z}^{\prime}=\frac{d m}{d z}, \Phi_{z}^{\prime}=\frac{d \Phi}{d z}$. Further, we assume a harmonic perturbation leading to inhomogeneous profiles $n=n(z), m=m(z)$ in eqs 26a-c

$$
m=\alpha_{m} \bar{n} \cos (q z), \quad n=\bar{n}\left(1+\alpha_{n} \cos (q z)\right)
$$

where we took into account that due to symmetry (with respect to reflection $z \rightarrow-z$ ) the phase shift between the polycation and polyanion phases is 0 or $\pi$. After substitution of $m=m(z)$, eq 27 , in eq $9 \mathrm{~b}$ and using expansion $\sinh \Phi \approx \Phi+\Phi^{3} / 6$ one gets

$$
\Phi(z) \simeq \frac{1}{2} \frac{\kappa \alpha_{m}}{1+q^{2} r_{D}^{2}} \cos (q z)-\frac{1}{64}\left(\frac{\kappa \alpha_{m}}{1+q^{2} r_{D}^{2}}\right)^{3}\left(\frac{3 \cos (q z)}{1+q^{2} r_{D}^{2}}+\frac{\cos (3 q z)}{1+9 q^{2} r_{D}^{2}}\right)
$$

Here we have introduced a new parameter $\kappa \equiv \bar{n} f / c_{s}$ : it is assumed that $\kappa \alpha_{m} \ll 1$ (cf. the discussion of the DH-approximation below eq 12). Substitution of eqs 27, 28 in the formulas 
26a-c (we put $\cosh \Phi-1 \approx \Phi^{2} / 2+\Phi^{4} / 24$ ) followed by an expansion of the free energy $\Delta \mathcal{F}$ with respect to $\alpha_{n}, \alpha_{m} \ll 1$ and integration yield

$$
\begin{aligned}
\frac{\Delta \mathcal{F}}{T \sqrt{n}} \simeq & -\frac{C \bar{n}^{2}}{3}+\frac{\alpha_{n}^{2}}{4}\left(\bar{n}^{2} C+\frac{a^{2} q^{2}}{2}\right)+\frac{\alpha_{m}^{2}}{8}\left[a^{2} q^{2}+\frac{\bar{n} B_{e s}}{1+q^{2} r_{D}^{2}}-\bar{n} \tilde{v}\right] \\
& +\frac{a^{2} q^{2}}{32}\left(\alpha_{m}^{4}+\alpha_{n}^{4}+6 \alpha_{m}^{2} \alpha_{n}^{2}\right)-\frac{5}{512} \frac{f \kappa^{3} \alpha_{m}^{4}}{\left(1+q^{2} r_{D}^{2}\right)^{4}}
\end{aligned}
$$

Obviously, the minimum of the free energy 29 is attained at $\alpha_{n}=0$. Further minimization of the second-order term $\sim \alpha_{m}^{2}$ with respect to wave-number $q$ shows that the homogeneous phase gets unstable if $\tilde{v}=B+v>B_{e s}$. This instability leads to a macroscopic phase separation (in $2 \mathrm{PE}$ phases) beyond the spinodal point $\tilde{v}=B_{e s}$ (i.e. at $\tilde{v}>B_{e s}$ ) if $X<1$, where

$$
X^{2}=\frac{r_{D}^{2} \bar{n} B_{e s}}{a^{2}}
$$

Note that

$$
X=c_{s}^{*} / c_{s}, \quad c_{s}^{*}=\frac{f}{a}\left(\frac{\bar{n}}{8 \pi l_{B}}\right)^{1 / 2} \simeq \frac{f}{a b^{2}}\left(\frac{\phi_{p}}{8 \pi u}\right)^{1 / 2}
$$

Note also that earlier studies of PE complexation and microphase separation used the inverse parameter $s=X^{-1}=c_{s} / c_{s}^{*} \cdot 14,15,32,33,41$

If $X \geq 1$ (that is, for $c_{s}^{*}>c_{s}$ ), the instability occurs as a microphase separation with the critical wave-number $q=q^{*}>0$,

$$
q^{*} r_{D}=\sqrt{X-1}
$$


The period of the lamellar structure at the spinodal is $\Lambda=2 \pi / q^{*}$. In order to locate this transition (for $X>1$ ), the free energy, eq 29, was written for $\alpha_{n}=0$ and $q=q^{*}$ :

$$
\frac{\Delta \mathcal{F}}{T V \bar{n}}=-\frac{C \bar{n}^{2}}{3}+\frac{a^{2} \alpha_{m}^{2}}{8 r_{D}^{2}}\left[2 X-1-X^{2} \frac{\tilde{v}}{B_{e s}}\right]+\frac{a^{2} \alpha_{m}^{4}}{32 r_{D}^{2} X^{3}}\left[X^{3}(X-1)-\frac{5}{16} \kappa^{2}\right]
$$

The lamellar phase formation occurs as the second order phase transition if the quartic term is positive, i.e., if

$$
X^{3}(X-1)>\frac{5}{16} \kappa^{2}
$$

In this case $X>1$ and the transition occurs at $\tilde{v}=\tilde{v}^{*}$,

$$
\tilde{v}^{*}=\frac{2 X-1}{X^{2}} B_{e s}=\left(1-\left(1-\frac{1}{X}\right)^{2}\right) B_{e s}
$$

The lamellar phase is stable beyond this critical point, i.e., for $\tilde{v}>\tilde{v}^{*}$. The last condition boils down to $\chi_{A C} \propto \tilde{v}>$ const $\left(2-c_{s} / c_{s}^{*}\right)$ in agreement with results of ref. 17 .

Slightly below the critical point the free energy 32 reads

$$
\frac{\Delta \mathcal{F}_{L}}{T V n} \simeq-\frac{C \bar{n}^{2}}{3}-\frac{\bar{n}}{16}\left(\tilde{v}-\tilde{v}^{*}\right) \alpha_{m}^{* 2}, \quad \alpha_{m}^{* 2}=\frac{2 X^{5}}{B_{e s}} \frac{\tilde{v}-\tilde{v}^{*}}{X^{3}(X-1)-\frac{5}{16} \kappa^{2}}
$$

Let us consider the regime of low enough salinity, $X \gg 1$. Assuming besides that $r_{D}^{2} f / a^{2} \gg 1$ (which means that the Debye length is larger than the typical distance between neighboring charged groups along the chain) we get a simplified expression for the amplitude: 


$$
\alpha_{m}^{*} \simeq \sqrt{\frac{2 X\left(\tilde{v}-\tilde{v}^{*}\right)}{B_{e s}}}, \quad \tilde{v}^{*} \simeq \frac{2}{X} B_{e s}
$$

leading to

$$
\frac{\Delta \mathcal{F}_{L}}{T V \bar{n}} \simeq-\frac{C \bar{n}^{2}}{3}-\frac{\bar{n} B_{e s} X}{8}\left(\frac{\tilde{v}}{B_{e s}}-\frac{2}{X}\right)^{2}
$$

The instability considered above results in formation of a weak lamellar structure. By changing parameters (like $\tilde{v})$ it can evolve into a strongly modulated coacervate phase (with $m \sim n)$. In the next section we analyze such structures formed in region III ${ }^{*}$. It is shown there that lamellar phases with strong segregation between PC and PA domains can also emerge in region II.

\section{Lamellar structures with well-defined domains}

Now we go beyond the perturbation scheme and consider lamellar structures with inhomogeneous composition, but uniform total concentration, i.e. $n=$ const and $\Delta \mathcal{F}_{2}=0$. Below we focus on the regime

$$
\kappa=n f / c_{s} \ll 1
$$

The latter condition is necessary for validity of the DH-approximation as long as $m \sim n$. Note that $\kappa / X=\sqrt{2 \tilde{l}_{B} a^{2} n}$, so the condition $\kappa \ll 1$ for $X \sim 1$ is equivalent to $\tilde{l}_{B} a^{2} n \ll 1$ which is consistent with the condition of validity for the virial expansion, $\phi_{p} \ll 1$, since $\tilde{l}_{B} a^{2} n \sim b^{3} n=\phi_{p}$.

Using non-dimensional variables 


$$
x=z / r_{D}, \quad y=m / n, \quad \tilde{\Phi}=\Phi /\left(f r_{D}^{2} \tilde{l}_{B} n\right)
$$

the free energy, eq 26a-c, at $|\tilde{\Phi}| \ll 1$ is written as

$$
\Delta \mathcal{F}=T V n\left[-\frac{1}{4}(B-v) n+\frac{C}{3} n^{2}+\frac{1}{4 \tilde{\Lambda}} \int_{-\tilde{\Lambda} / 2}^{\tilde{n} / 2}\left(\frac{a^{2}}{r_{D}^{2}} \frac{y_{x}^{\prime 2}}{1-y^{2}}-n B_{e s}\left(\tilde{\Phi}^{2}+\tilde{\Phi}_{x}^{\prime 2}-2 y \tilde{\Phi}\right)-n \tilde{v} y^{2}\right) d x\right]
$$

where $y_{x}^{\prime}=\frac{d y}{d x}, \tilde{\Phi}_{x}^{\prime}=\frac{d \tilde{\Phi}}{d x}, \tilde{\Lambda}=\Lambda / r_{D}$ and $\Lambda$ is the period of the lamellar structure. Equation $9 \mathrm{~b}$ can be rewritten in a simple form

$$
\tilde{\Phi}_{x x}^{\prime \prime}=\tilde{\Phi}-y
$$

where $\tilde{\Phi}_{x x}^{\prime \prime}=\frac{d^{2} \tilde{\Phi}}{d x^{2}}$

The theory can be simplified in the regime of strong segregation when the interfacial layer thickness $\Delta_{i}$ between polyanion and polycation domains is much smaller than the period of the lamellar structure, $\Lambda, \Delta_{i} \ll \Lambda$. In this case we can use the strong segregation approximation: the free energy, eq 40, can be presented as a sum of the short range interaction energy $\mathcal{F}_{\text {int }}$ inside domains, the interfacial energy between domains $\mathcal{F}_{\text {inf }}$ and the long-range electrostatic energy $\mathcal{F}_{\text {es }}:$

$$
\Delta \mathcal{F}=\mathcal{F}_{\text {int }}+\mathcal{F}_{\text {inf }}+\mathcal{F}_{\text {es }}
$$

The interaction energy is given by 


$$
\mathcal{F}_{\text {int }}=T V\left[-\frac{B}{2} n^{2}+\frac{C}{3} n^{3}\right]
$$

The interfacial energy can be presented as

$$
\mathcal{F}_{\text {inf }} \simeq T V \frac{n}{2 \tilde{\Lambda}} \int_{-\infty}^{\infty}\left(\frac{a^{2}}{r_{D}^{2}} \frac{y_{x}^{\prime 2}}{1-y^{2}}+n \tilde{v}\left(1-y^{2}\right)\right) d x
$$

In the last formula the boundary conditions reflecting strong segregation between the domains are assumed: $y(-\infty)=1$ and $y(\infty)=-1$. Minimization of eq 44 yields the profile

$$
y(x)=\tanh \left(r_{D} x / \Delta_{i}\right)
$$

where the interfacial layer thickness is

$$
\Delta_{i}=a / \sqrt{n \tilde{v}}
$$

Therefrom we get (for $\Delta_{i} \ll \Lambda$ )

$$
\mathcal{F}_{\text {inf }} \simeq 2 T V \frac{a n^{3 / 2} \sqrt{\tilde{v}}}{r_{D} \tilde{\Lambda}}
$$

The corresponding interfacial tension is $\gamma_{i} \simeq \operatorname{Tn}^{2} \tilde{v} \Delta_{i}$.

Finally, the electrostatic energy within the DH-approximation is written as

$$
\mathcal{F}_{\text {es }}=T V \frac{B_{e s} n^{2}}{2 \tilde{\Lambda}} \int_{-\tilde{\Lambda} / 4}^{\tilde{\Lambda} / 4}\left(2 y \tilde{\Phi}-\tilde{\Phi}^{2}-\tilde{\Phi}_{x}^{\prime 2}\right) d x
$$

The long-range character of electrostatic interactions allows to use a step-like profile (since $\left.\Delta_{i} \ll r_{D}, \Delta_{i} \ll \Lambda\right):$ 


$$
y(x) \approx\left[\begin{array}{lc}
1, & -\tilde{\Lambda} / 4<x<0 \\
-1, & 0<x<\tilde{\Lambda} / 4
\end{array}\right.
$$

This function is defined for a half period of the lamellar structure with the interface located at $z=0 . y(x)$ is extended to the whole range $-\infty<x<\infty$ using reflection symmetry at $x= \pm \tilde{\Lambda} / 4$.

The continuous potential $\tilde{\Phi}(x)$ is defined by eq 41 using additional conditions $\tilde{\Phi}(-\tilde{\Lambda} / 4)=-\tilde{\Phi}(\tilde{\Lambda} / 4), \tilde{\Phi}_{x}^{\prime}(-\tilde{\Lambda} / 4)=\tilde{\Phi}_{x}^{\prime}(\tilde{\Lambda} / 4)=0$. Thus we get:

$$
\tilde{\Phi}(x) \simeq\left[\begin{array}{ll}
1-\frac{e^{-x}+e^{x+\tilde{\Lambda} / 2}}{e^{\tilde{\Lambda} / 2}+1}, & -\tilde{\Lambda} / 4<x<0 \\
-1+\frac{e^{-x}+e^{x-\tilde{\Lambda} / 2}}{e^{-\tilde{\Lambda} / 2}+1}, & 0<x<\tilde{\Lambda} / 4
\end{array}\right.
$$

The maximum potential value is $\tilde{\Phi}^{*}=2 \sinh ^{2}(\tilde{\Lambda} / 8) / \cosh (\tilde{\Lambda} / 4)$ and the electric field strength at the interface is $\left|\tilde{\Phi}_{x}^{\prime}(0)\right|=\tanh (\tilde{\Lambda} / 4)$. Substitution of eq 49a in eq 47 yields

$$
\mathcal{F}_{\text {es }}=T V \frac{B_{e s} n^{2}}{4}\left(1-\frac{4}{\tilde{\Lambda}} \tanh \left(\frac{\tilde{\Lambda}}{4}\right)\right)
$$

The total free energy then becomes

$$
\frac{\Delta \mathcal{F}}{T V n}=-\frac{1}{2}\left(B-\frac{B_{e s}}{2}\right) n+\frac{C}{3} n^{2}+\frac{B_{e s} n}{\tilde{\Lambda}}\left(\beta-\tanh \left(\frac{\tilde{\Lambda}}{4}\right)\right)
$$

where

$$
\beta=\frac{2 a \sqrt{\tilde{v}}}{r_{D} B_{e s} \sqrt{n}}=\frac{2 \tilde{v} \tilde{\Delta}}{B_{e s}}=\frac{2}{X} \sqrt{\frac{\tilde{v}}{B_{e s}}}
$$


It is obvious that for $\beta \geq 1$ the minimum of this free energy is attained at $\tilde{\Lambda}=\infty$ corresponding to a uniform macro-phase. By contrast, a lamellar structure is stable for $\beta<1$. Minimization of the free energy, eq 51a, with respect to $\tilde{\Lambda}$ gives the following equation for the period of the lamellar structure

$$
\beta-\tanh \left(\frac{\tilde{\Lambda}}{4}\right)+\frac{\tilde{\Lambda}}{4 \cosh ^{2}(\tilde{\Lambda} / 4)}=0
$$

The transition from a finite to infinite period $\tilde{\Lambda}$ for a mixture of weakly charged and neutral polymers was predicted and discussed in ref. 21 . The total polymer concentration in the lamellar phase can be found by minimization of $\Delta \mathcal{F}$ with respect to $n$ at $n V=$ const (this condition is equivalent to setting osmotic pressure $\Pi=n^{2} \frac{\partial}{\partial n}\left(\frac{\Delta \mathcal{F}}{n V}\right)$ to zero):

$$
\frac{\partial}{\partial n}\left(\frac{\Delta \mathcal{F}}{n V}\right)=0
$$

After substitution of the free energy eq 51a in eq 53 and using eq 52 one gets

$$
n=n_{L}=\frac{3 B}{4 C}\left[1-\frac{B_{e s}}{2 B} \tanh ^{2}\left(\frac{\tilde{\Lambda}}{4}\right)\right]
$$

The total free energy, eq 51a, can be written in terms of $n_{L}$ :

$$
\frac{\Delta \mathcal{F}}{T V n}=-\frac{1}{2}\left(B-\frac{B_{e s}}{2} \tanh ^{2}\left(\frac{\tilde{\Lambda}}{4}\right)\right) n+\frac{C}{3} n^{2}=-\frac{C}{3} n_{L}^{2}
$$

The lamellar phase is stable if the free energy 55 is smaller than the free energy of the isotropic phases in the regions II or III". This criterion can be reformulated in terms of polymer 
concentration since in all the cases the free energy is $\Delta \mathcal{F} /(T V n)=-C n^{2} / 3$. Hence the phase with higher polymer concentration is always more stable. Obviously, $n_{L} \geq n_{I I}=\frac{3 B}{4 C}\left[1-\frac{B_{e s}}{2 B}\right]$ (cf. eq 14), therefore it seems that the lamellar phase is always more favorable thermodynamically in the region II provided that $\beta<1$. Recalling that this regime implies that $\tilde{v}>B_{e s}$ (cf. Figure 1), we arrive at the following subregion $\mathrm{II}_{\mathrm{L}}$ of lamellar structure stability:

$$
1<\frac{\tilde{v}}{B_{e s}}<\frac{X^{2}}{4}
$$

Some clarifications are required at this point: (i) The above criterion applies to the strongly segregated structures only. (ii) It must be supplemented by the conditions of validity of the narrow interface approximation adopted here:

$$
r_{D} \gg \Delta_{i}, \quad \Lambda \gg \Delta_{i}
$$

From eqs $45 \mathrm{~b}, 51 \mathrm{~b}$ we get

$$
\frac{\Delta_{i}}{r_{D}}=\frac{\beta}{2} \frac{B_{e s}}{\tilde{v}}=\frac{1}{X} \sqrt{\frac{B_{e s}}{\tilde{v}}}
$$

The condition $\frac{\Delta_{i}}{r_{D}}=\frac{1}{X} \sqrt{\frac{B_{e s}}{\tilde{v}}} \ll 1$ together with $\beta=\frac{2}{X} \sqrt{\frac{\tilde{v}}{B_{e s}}}<1$ implies that $X^{2} \gg 1$. By virtue of eq 52 the latter condition also insures that $\Lambda \gg \Delta_{i}$. Therefore, the condition $X \gg 1$ must be added to the conditions 56 .

In the general case of any positive ratio $\tilde{v} / B_{e s}$ (including the regime III*) the condition $\Lambda \gg \Delta_{i}$ is satisfied if $B_{e s} / \tilde{v} \ll X$. It is easy to show (using eq 55) that the latter condition 
(together with $X \gg 1$ ) is sufficient to stabilize the lamellar phase in region III ${ }^{*}$. Therefore, the whole region of the strongly segregated lamellar structure is defined by conditions

$$
1 / X \ll \tilde{v} / B_{e s}<X^{2} / 4, \quad X \gg 1
$$

The period of the lamellar structure and the polymer concentration can be found analytically in the limiting cases of small and large $\tilde{\Lambda}$. First we assume that $\tilde{\Lambda} \ll 1(\beta \ll 1)$. From eqs 52, 54 we get:

$$
\begin{gathered}
\Lambda \simeq 4 r_{D}(1.5 \beta)^{1 / 3} \simeq 2\left(\frac{3}{\pi}\right)^{1 / 3}\left(\frac{a}{l_{B}}\right)^{1 / 3} \frac{\tilde{v}^{1 / 6}}{f^{2 / 3} n^{1 / 6}} \\
n=n_{L} \simeq \frac{3 B}{4 C}\left[1-\frac{B_{e s}}{2 B}(1.5 \beta)^{2 / 3}\right]
\end{gathered}
$$

Note that the period is independent of salinity $c_{s}$ and scales as $\Lambda \sim a \tilde{v}^{1 / 6} f^{-2 / 3}$ in agreement with the previous finding. ${ }^{23}$ The stability condition 59 together with $\beta \ll 1$ are equivalent to $1 / X \ll \tilde{v} / B_{e s} \ll X^{2} / 4, X \gg 1$. The free energy of the lamellar phase in this case reads

$$
\frac{\Delta \mathcal{F}}{T V \bar{n}} \simeq-\frac{C \bar{n}^{2}}{3}-\frac{\bar{n} B_{e s}}{4}\left(\frac{\tilde{v}}{B_{e s}}-\frac{3}{X}\right)-\frac{3 B_{e s}^{2}}{64 C}\left(\frac{\tilde{v}}{B_{e s}}-\frac{3}{X}\right)^{2}
$$

Another limiting case corresponds to $\tilde{\Lambda} \gg 1(1-\beta \ll 1)$. Equations 52,54 now produce the following results

$$
\Lambda \simeq 2 r_{D} \ln \left(\frac{1}{1-\beta}\right), \quad n=n_{L} \simeq \frac{3 B}{4 C}\left[1-\frac{B_{e s}}{2 B}(4 \beta-3)\right]
$$

Stabilization of the long-period lamellar phase occurs near the boundary $\tilde{v} / B_{e s} \approx X^{2} / 4, X \gg 1$. 
In order for the strongly-segregated lamellar phase to be stable, it is necessary to fulfill the conditions $X \gg 1, \kappa \ll 1, \beta<1$. They lead to

$$
\begin{aligned}
& r_{D}^{2} \gg \frac{a}{f}\left(2 \tilde{l}_{B} n\right)^{-1 / 2} \\
& r_{D}^{2} \ll\left(2 \tilde{l}_{B} f n\right)^{-1} \\
& r_{D}^{3}>\frac{a}{2 \tilde{l}_{B} f^{2}} \sqrt{\frac{\tilde{v}}{n}}
\end{aligned}
$$

These conditions are compatible if

$$
\begin{aligned}
& a \sqrt{\tilde{l}_{B} n} \ll 1 \\
& f \gg \tilde{l}_{B} a^{2} n^{2} \tilde{v}
\end{aligned}
$$

Note that lamellar structures emerge in solution of soluble polyelectrolytes if an additional condition $B_{e s}>2 B$ is satisfied implying that $\phi_{p}^{2}<f \kappa$. The latter condition with $\kappa \ll 1$ ensures validity of condition $65 \mathrm{~b}$.

\section{General conditions for the lamellar phase stability}

As shown above, the strongly segregated lamellar structures are stable for $X \gg 1$. It is also clear that a domain structure cannot possibly be favorable for $X \rightarrow 0$ since electrostatic contribution is totally suppressed in this limit $\left(r_{D} \rightarrow 0\right)$. Therefore there must be a minimal $X=X_{c}$ compatible with lamellar phase stability. It is evaluated below. 
Note that the condition $X \gg 1$ comes, in particular, from the requirement of long-range electrostatics, $r_{D} \gg \Delta_{i}$, since $X$ is proportional to $r_{D}^{2}: X=r_{D}^{2}(f / a) \sqrt{2 \tilde{l}_{B} n}$. Below we show that an opposite regime (allowing for a lower $X$ ), $r_{D} \ll \Delta_{i}$, is relevant in regime II close to its boundary with regime III $^{*}$. In this case the electrostatic interactions are relatively short-range and mainly lead to a renormalization of the second virial coefficients providing a term $\propto B_{e s} m^{2}$ in the free energy density (cf. eq 12). It is important, however, to include the gradient correction to this term coming from the $q$-dependence of $B_{e s}$ :

$$
B_{e s} \rightarrow B_{e s}^{*}=\frac{B_{e s}}{1+q^{2} r_{D}^{2}} \simeq B_{e s}\left(1-q^{2} r_{D}^{2}\right), \quad q r_{D} \ll 1
$$

Taking this correction into account leads to the following expression for the electrostatic free energy (assuming as before that the total concentration $n$ is uniform):

$$
\mathcal{F}_{e s} \simeq T\left(B_{e s} / 4\right) n^{2} \int_{V}\left[y^{2}-r_{D}^{2} y_{z}^{\prime 2}\right] d V
$$

The same result can be obtained directly from eqs 47,41 for a smooth charge density profile with characteristic length-scale $\gg r_{D}$. The total interfacial tension between 2 homogeneous macro-phases with $|y| \rightarrow 1$ therefore is (cf. eqs. (44), (67), (12)):

$$
\gamma_{\mathrm{inf}} \simeq \min _{y(z)} \int_{-\infty}^{\infty} d z\left\{\frac{n^{2}}{4}\left(\tilde{v}-B_{e s}\right)\left(1-y^{2}\right)+\frac{n a^{2}}{4\left(1-y^{2}\right)} y_{z}^{\prime 2}-\frac{B_{e s} n^{2} r_{D}^{2}}{4} y_{z}^{\prime 2}\right\}
$$

Let us first suppress the last term in curly brackets. In this case the result is

$$
y_{0}(z)=\tanh \left(z / \Delta_{i 0}\right), \quad \Delta_{i 0}=\frac{a}{\sqrt{n\left(\tilde{v}-B_{e s}\right)}}
$$


and

$$
\gamma_{\text {inf } 0}=\operatorname{Tan}^{3 / 2}\left(\tilde{v}-B_{e s}\right)^{1 / 2}
$$

This tension is positive if $\tilde{v}>B_{e s}$. Can the full $\gamma_{\text {inf }}$ (including the last negative term) become negative? The answer is certainly yes if $\tilde{v}-B_{e s} \ll B_{e s}$. The simplest case is $\tilde{v}=B_{e s}$ : then only the gradient terms are present in eq 68. For $n a^{2}>B_{e s} n^{2} r_{D}^{2}$, i.e. $X<1$, the integrand there is necessarily positive, hence $\gamma_{\mathrm{inf}}>0$ again. However, as soon as $X$ exceeds 1 minimization in eq 68 always leads to $\gamma_{\text {inf }}<0$. The condition $q r_{D} \ll 1$ is not a problem since for $\tilde{v} / B_{e s}=1$ the r.h.s. of eq 68 does not change sign (remains negative) for however smooth profile $y(z)$. Similarly, $\gamma_{\text {inf }}$ remains negative also for $\tilde{v} / B_{e s}=1+\varepsilon, \varepsilon \ll 1$ if $X=1+\delta$, with some $\delta \ll 1$.

Negative $\gamma_{\text {inf }}$ means that interfaces are favorable, so a domain (lamellar) structure with period $\Lambda \gg \Delta_{i}$ must be more favorable than 2 coexisting macro-phases (a similar idea was proposed in ref. 21). Hence we arrive at $X_{c}=1$ and predict that lamellar structures are stable in a part of region II, for

$$
1<\tilde{v} / B_{e s}<r^{*}(X), \quad X>1
$$

where $r^{*}(1)=1$. This conclusion is in agreement with eq 56 which is valid for $X \gg 1$. It shows that

$$
r^{*}(X) \approx X^{2} / 4, \quad X \gg 1
$$

Combining the above results we propose the following extrapolation: 


$$
r^{*}(X) \approx 1+(X-1)^{2} / 4, \quad X>1
$$

What about the single-phase region III $^{*}$ where $\tilde{v}<B_{e s}$. The analysis of section 4 shows that a lamellar structure is expected for (cf. eq 34):

$$
\frac{2 X-1}{X^{2}}<\tilde{v} / B_{e s}<1, \quad X>1
$$

As before we assume that $\kappa \ll 1$ (cf. eq 38); note that eq 33 with $\kappa \ll 1$ is virtually equivalent to $X>1$. The above inequalities are obviously in harmony with eq 59 which is valid in the regime of strong segregation.

Remarkably we thus arrived at the same condition of a lamellar phase stability $(X>1$ for $\tilde{v} / B_{e s} \approx 1$ ) using 2 entirely different approaches: in one case (cf. eq 31-34) a nearly uniform phase with a weak harmonic composition profile was considered at $\tilde{v} / B_{e s}<1$. By contrast, in the current section we considered a strongly segregated domain structure with maximum composition contrast between the domains (from $p \approx 1$ to $p \approx-1$ ) and thin interfaces $\left(\Delta_{i} \ll \Lambda\right.$ ) for $\tilde{v} / B_{e s}$ slightly exceeding 1 . It is therefore reasonable to expect that the nature of lamellar structure changes from weak to strong as the ratio $\tilde{v} / B_{e s}$ increases from $\tilde{v} / B_{e s}<1$ (regime III*) to $\tilde{v} / B_{e s}>1$ (regime II) at $X>1$.

The schematic phase diagram in coordinates $X, r=\tilde{v} / B_{e s}$ is shown in Figure 2 for $2 B>\tilde{v}$. It includes regions of 1 uniform phase $\left(r<1, X<1\right.$ and $\left.r<\frac{2 X-1}{X^{2}}, X>1\right)$, of 2 coexisting uniform phases $\left(r>1, X<1\right.$ and $\left.r>r^{*}(X), X>1\right)$, and of lamellar phase $\left(r^{*}(X)>r>\frac{2 X-1}{X^{2}}, X>1\right)$. The critical (Lifshitz) point is located at $r=1, X=1$. This 
prediction is in agreement with the earlier results on microphase separation in PEs ${ }^{14,15,41,43}$ revealing a Lifshitz point located at $s=1 / X=1$.

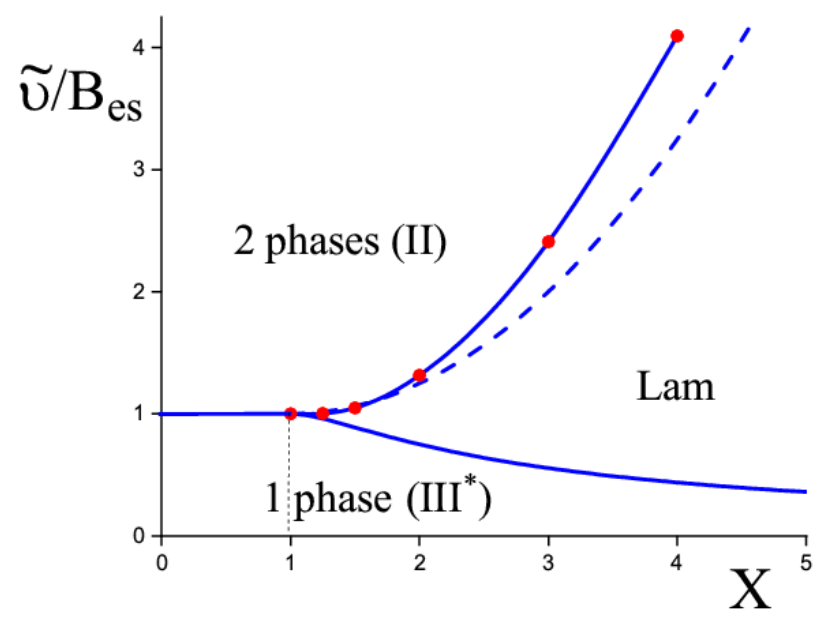

Figure 2. Phase diagram in coordinates $\left(X, r=\tilde{v} / B_{e s}\right)$. The dashed line is obtained from the eq (71b). Filled circles are from numerical calculations, see section 7 . The solid line passed through the circles is the boundary between the 2 phase region II and Lam phase. The solid line between the 1 phase region III $^{*}$ and Lam phase is obtained from the eq 34.

Note that a qualitatively similar phase diagram has been proposed in ref. 43 based on the weak segregation approximation (which was implied by their RPA approach). The theory of ref. 43 thus yields the same equation $\tilde{v}=B_{e s}$ for the boundaries II/III ${ }^{*}$ and II/Lam. Our study shows, however, that the RPA approximation is never applicable near the boundary Lam/II between microphase separated coacervate (Lam) and the two-macrophase region (II), apart from the Lifshitz point. As demonstrated in section 5 the microdomain structure near the Lam/II boundary 
is strongly segregated. Our quantitative theory (see section 7) is more general and is valid for both weak and strong segregation regimes. It shows that the Lam/II boundary is located strongly above the line $\tilde{v}=B_{e s}$ in low-salt conditions, $X \gg 1$ (cf. Figure 2). It may be also worth mentioning that the phase diagram of Figure 2 is universal for the virial model we consider.

\section{Numerical analysis of the lamellar structure}

Minimization of the free energy (40) with respect to $y(x)$ yields

$$
\frac{y_{x x}^{\prime \prime}}{1-y^{2}}+\frac{y y_{x}^{\prime 2}}{\left(1-y^{2}\right)^{2}}=X^{2}\left(\tilde{\Phi}-\frac{\tilde{v}}{B_{e s}} y\right)
$$

Here $y_{x x}^{\prime \prime}=\frac{d^{2} y}{d x^{2}}$. Integration of eqs 41,73 results in the following conservation law

$$
-\frac{y_{x}^{\prime 2}}{1-y^{2}}+X^{2}\left(\tilde{\Phi}_{x}^{\prime 2}+2 y \tilde{\Phi}-\tilde{\Phi}^{2}-\frac{\tilde{v}}{B_{e s}} y^{2}\right)=X^{2} I=\text { const }
$$

where $X^{2} I$ is integration constant. The constant $I$ can be expressed through the maximum values $\tilde{\Phi}^{*}$ and $y^{*}$ of the periodic functions $\tilde{\Phi}(x)$ and $y(x)$ (note, by symmetry these values are attained in the middle of the domains where $\left.\tilde{\Phi}_{x}^{\prime}=0, y_{x}^{\prime}=0\right)$ :

$$
I=2 y^{*} \tilde{\Phi}^{*}-\tilde{\Phi}^{* 2}-\frac{\tilde{v}}{B_{e s}} y^{* 2}
$$

The free energy 40 after using eq 74a is written as

$$
\frac{\Delta \mathcal{F}}{T V n}=-\frac{1}{4}(B-v) n+\frac{C}{3} n^{2}+\frac{1}{2} n B_{e s} J,
$$




$$
J=\frac{1}{\tilde{\Lambda}} \int_{-\tilde{\Lambda} / 2}^{\tilde{\Lambda} / 2}\left[2 y \tilde{\Phi}-\tilde{\Phi}^{2}-\frac{\tilde{v}}{B_{e s}} y^{2}-\frac{I}{2}\right] d x
$$

The lamellar structure profile and the period for a given polymer concentration $n$ is found from numerical solution of equations 41 and 73 treating $y^{*}$ and $\tilde{\Phi}^{*}$ as fitting parameters. To do this, we set the value of $y^{*}$ and numerically solve the system of eqs 41,73 by choosing the desired value of $\tilde{\Phi}^{*}$ to get half-period of the lamellar structure with knots $(y=0$ and $\Phi=0)$ at the same point $x$. Further, the function $J\left(y^{*}\right)$ is found for the obtained profiles using eq $75 b$. Since $\tilde{\Phi}^{*}$ enters eqs $74 \mathrm{a}, \mathrm{b}$ quadratically, one value of $y^{*}$ in the general case may correspond to two different values of $\tilde{\Phi}^{*}$. Therefore, two different profiles $\tilde{\Phi}(x)$ and $y(x)$ may exist for a given $y^{*}$, so that the function $J\left(y^{*}\right)$ can have two branches (with two values of $J\left(y^{*}\right)$ corresponding to the same $y^{*}$ ). The optimum lamellar structure is obtained by minimization of $J\left(y^{*}\right)$ with respect to the amplitude $y^{*}$. The dependence of $J\left(y^{*}\right)$ on the amplitude $y^{*}$ for $X=1.5, \tilde{v} / B_{e s}=0.95 ; 1.0$ and $X=2, \tilde{v} / B_{e s}=0.9 ; 1.0$ are shown in Figure $3 \mathrm{a}, \mathrm{b}$ and the optimum lamellar structures corresponding to the minimum of $J\left(y^{*}\right)$ are shown in Figure $4 \mathrm{a}, \mathrm{b}$ and Figure 5a,b.
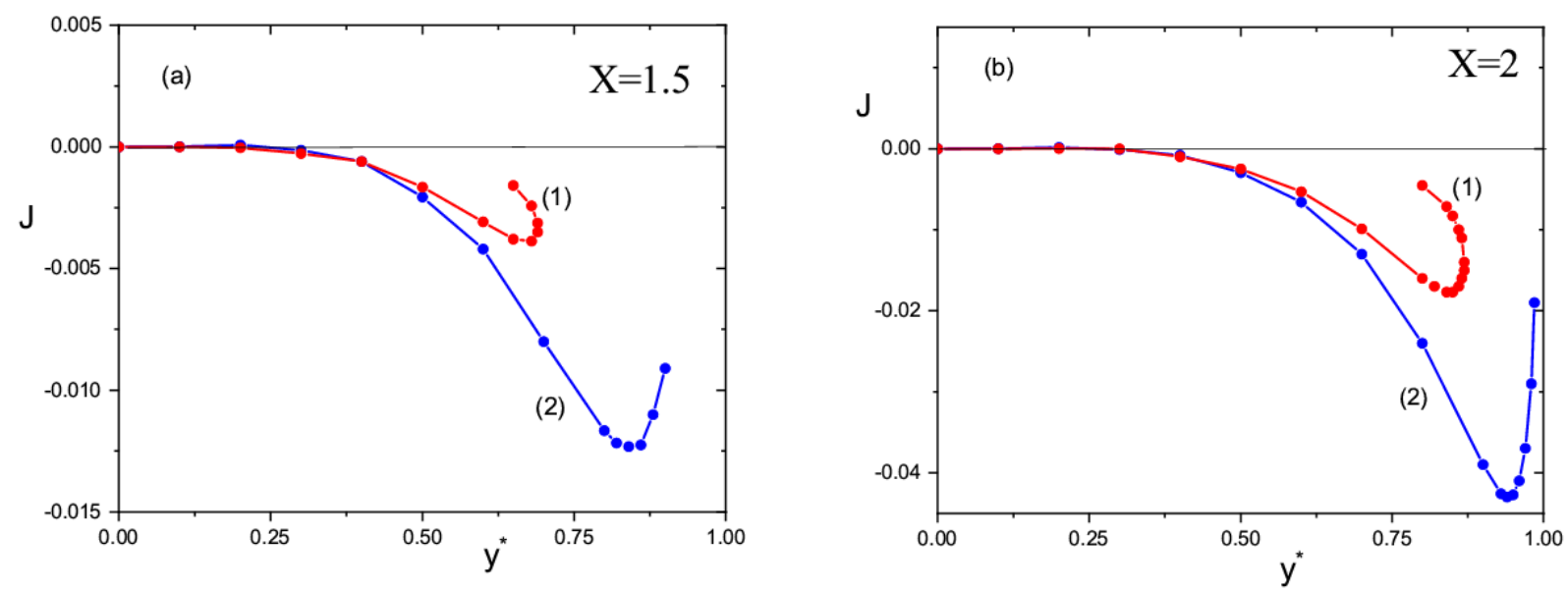
Figure 3. Dependence of the parameter $J$ on the amplitude $y^{*}$. (a): $X=1.5$, curve (1) corresponds to $\tilde{v} / B_{e s}=0.95$ and curve (2) $-\tilde{v} / B_{e s}=1$, (b): $X=2$, curve (1) corresponds to $\tilde{v} / B_{e s}=0.9$, curve (2) $-\tilde{v} / B_{e s}=1$.
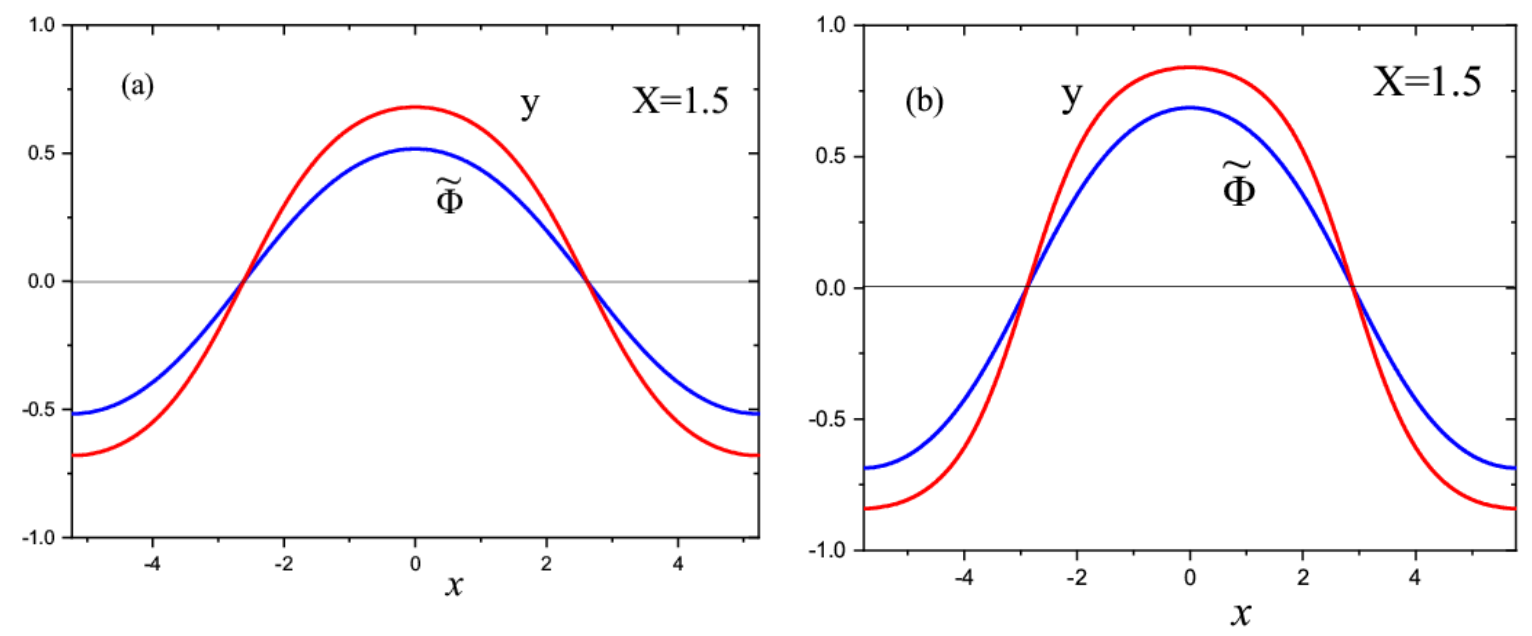

Figure 4. Profiles of the lamellar structure $y(x)$ and electric potential $\tilde{\Phi}(x)$ at $X=1.5$ and (a) $\tilde{v} / B_{e s}=0.95\left(\tilde{\Lambda} \approx 10.5, J_{\min } \approx-0.0039\right)$, (b) $\tilde{v} / B_{e s}=1\left(\tilde{\Lambda} \approx 11.5, J_{\min } \approx-0.012\right)$.
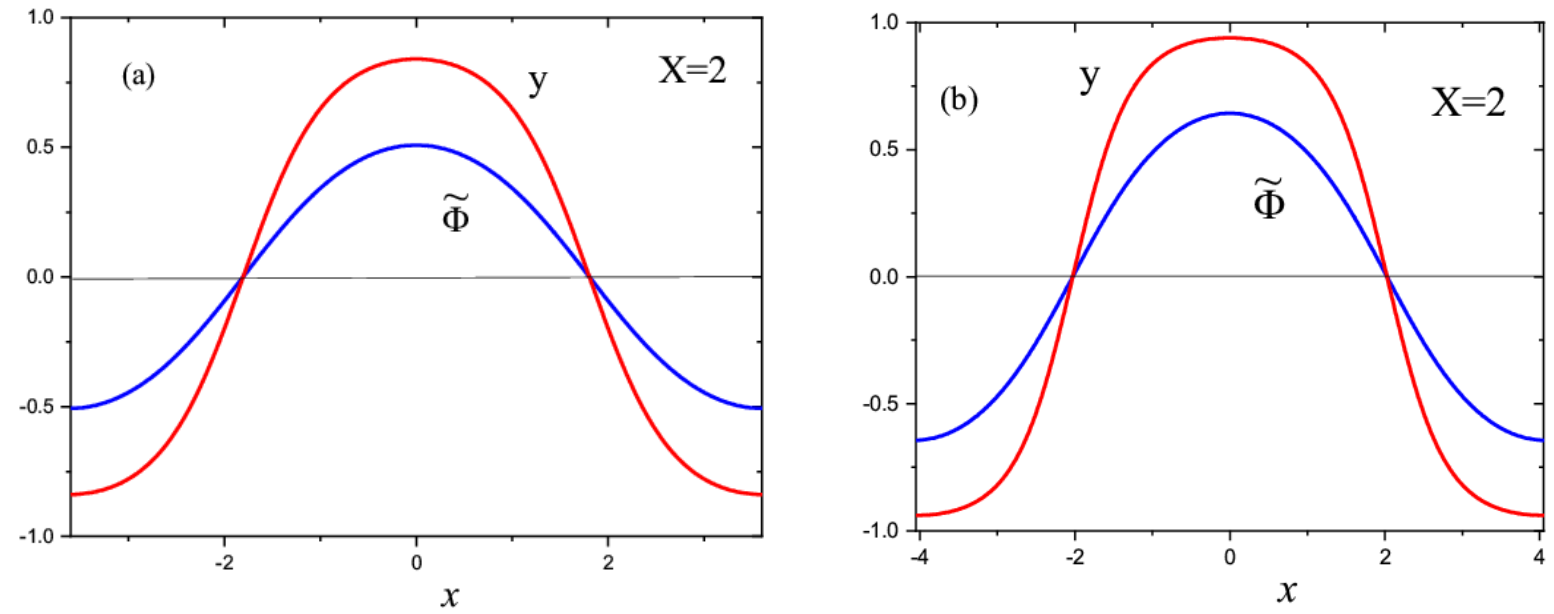
Figure 5. Profiles of the lamellar structure $y(x)$ and electric potential $\tilde{\Phi}(x)$ at $X=2$ and (a) $\tilde{v} / B_{e s}=0.9\left(\tilde{\Lambda} \approx 7, J_{\text {min }} \approx-0.018\right),\left(\right.$ b) $\tilde{v} / B_{e s}=1\left(\tilde{\Lambda} \approx 8, J_{\text {min }} \approx-0.043\right)$.

With increasing $\tilde{v} / B_{e s}$, the composition inhomogeneity of the lamellar phase also increases. The boundary $r^{*}(X)$ between the lamellar phase (region "Lam" in Figure 2) and 2 homogeneous phases (region II) is defined by the condition that their free energies per particle, $\frac{\Delta \mathcal{F}}{T V n}$, are equal. On using eq 75a and eq 12 with $m=n$ the latter condition converts to $2 J=1-\frac{\tilde{v}}{B_{e s}}$.

The polymer concentration $n$ can be obtained using the condition of zero osmotic pressure, $\Pi=0$ (cf. eq 53). Substitution of the free energy 75a,b in eq 53 yields

$$
n=\frac{3}{8 C}\left[B-\tilde{v}-2 J B_{e s}\right]
$$

(The derivative in eq 53 is taken at constant profiles $y(x), \tilde{\Phi}(x)$, hence $J=$ const as well.) Thermodynamic stability of the lamellar phase implies that its concentration $n$, eq 76, is higher than concentrations of the homogeneous phases $\bar{n}$ and $n_{I I}$ (cf. eqs 14, 25). These criteria lead to the conditions $J<0$ and $2 J<1-\frac{\tilde{v}}{B_{e s}}$, coming from competition with homogeneous coacervate phase and pure PE phases, respectively. The general conditions of lamellar structure stability therefore read

$$
2 J<\min \left(0,1-\frac{\tilde{v}}{B_{e s}}\right)
$$


The thermodynamic transition line, $r^{*}(X)$, between the lamellar (Lam) phase and the two-phase region (II) was obtained using the criterion $\gamma_{\text {inf }}=0$ following the ideas described in section 6. Here $\gamma_{\text {inf }}$ is the interfacial tension between two homogeneous PE phases coexisting in region II. The tension is defined in eq 68, which can be transformed (using eqs $74 a, b$ ) to

$$
\gamma_{\mathrm{inf}}=\operatorname{Tn}^{2} B_{e s} r_{D} \Gamma
$$

where

$$
\Gamma=\frac{1}{2} \int_{-\infty}^{\infty}\left[(r-1)\left(1-y^{2}\right)-(\Phi-y)^{2}\right] d x
$$

The functions $y(x)$ and $\Phi(x)$ were found by solving eqs 41 and 74 with boundary conditions $y=\Phi=1$ at $x=\infty$ and $y=\Phi=-1$ at $x=-\infty$, leading to

$$
I=1-r, \quad r \equiv \tilde{v} / B_{e s}
$$

The asymptotics of $\Gamma$ for high/low $X$ (obtained using the theory of sections 5,6) are

$$
\Gamma \simeq \begin{cases}\sqrt{r} / X-1 / 2, & X \gg 1 \\ \sqrt{r-1} / X, & X \ll 1\end{cases}
$$

The obtained results, which are shown in Figure 2, are in agreement with the low-salt asymptotic behavior given in eq 71a. Note that the first line in eq. (80) gives

$$
\gamma_{\mathrm{inf}}=\operatorname{Tan}^{3 / 2} \sqrt{\tilde{v}}-0.5 \operatorname{Tn}^{2} B_{e s} r_{D}
$$

where the first term is due to interactions of neutral polymer segments, while the second term comes from electrostatic interactions. The electrostatic term and the general structure of $\gamma_{\text {inf }}$ in 
the strong segregation regime had been considered in ref. 21. It is also worth noting that we demonstrated here (both analytically and numerically) that the interfacial thickness $\Delta_{\text {inf }}$ remains finite at the transition line, where $\gamma_{\mathrm{inf}}=0$ :

$$
\Delta_{\mathrm{inf}} \sim \frac{a}{\sqrt{n\left(\tilde{v}-B_{e s}\right)}}
$$

The above equation is valid for any $\tilde{v}>B_{e s}$, and, in particular, both in the weak segregation regime (where $\Delta_{\text {inf }} \gg r_{D}$ ) and for strong segregation (where $\Delta_{\text {inf }} \ll r_{D}$ ).

\section{Adsorbed multilayer}

In this section we study formation of polyelectrolyte multilayer on a charged surface upon the layer-by-layer deposition process. This process involves alternating contact of the surface multilayer with bulk solution containing soluble polyions carrying a charge opposite to the charge of the chains used in the previous stage of the multilayer formation. After a sufficient deposition time some part of the chains penetrate inside the multilayer. Any non-adsorbed chains are then removed by washing with clear water solution.

When forming a layer, two scenarios can be distinguished. The first scenario is realized for compatible soluble polyions (cf. the filled triangle area in Figure 1b defined by conditions $-B<v<B$ and $B_{e s}>2 B$ ). The second case corresponds to soluble polyions forming lamellar structures, implying that $X>1$, cf. Figure 2. Below we focus on the first case when the multilayer has a nearly homogeneous structure. Let us consider a negatively charged surface with charge density $-e \sigma$ and assume that during the deposition time the incoming from the outer 
solution chains can diffuse through the whole multilayer and thus can establish thermodynamic equilibrium between the multilayer and the bulk solution (as long as the oppositely charged polyions remain trapped in the layer, cf. eq. 85 below). Let $L_{k}$ be the layer thickness after $k$ deposition steps. We consider the regime of sufficiently thick layer, $L_{k} \gg \xi$, where $\xi=\max \left(r_{D}, \Delta_{i}\right)$ is the characteristic correlation length defining the thickness of the proximal sublayer, where the charge density is inhomogeneous due to the adsorbing surface effect ( $\Delta_{i}$ is defined in eq $45 b)$.

Generally the equilibrium composition of the adsorbed layer depends on the chemical potentials $\mu^{*}$ of bulk polyions and the bulk osmotic pressure. We assume that concentration $n_{b}$ of polyions in the bulk is very small (dilute solution regime), so that the bulk osmotic pressure is mainly defined by the ideal gas term and is negligible, $\Pi_{b} \simeq \operatorname{Tn}_{b} / N \approx 0$. The chemical potential of polymer segments is $\mu^{*} \simeq(T / N) \ln n_{b}$. Equilibrium concentration of polymer segments inside a thick adsorbed layer far away from the surface and the free interface can be found using eq 13 as explained below.

Suppose that the bulk contains polycations. The amount of polyanions inside the layer is fixed and defined in the previous stage of the deposition process. The chemical potentials of polycations $\mu_{C}$ and polyanions $\mu_{A}$ in the layer can be found using the free energy, eq 12, supplemented with the ideal-gas contribution:

$$
\begin{aligned}
& \mu_{C} / T=\frac{1}{N} \ln n_{C}-\frac{1}{2}(B-v) n+C n^{2}+\frac{p}{2}\left(B_{e s}-\tilde{v}\right) n \\
& \mu_{A} / T=\frac{1}{N} \ln n_{A}-\frac{1}{2}(B-v) n+C n^{2}-\frac{p}{2}\left(B_{e s}-\tilde{v}\right) n
\end{aligned}
$$


where

$$
n_{C}=\frac{1}{2} n(1+p), \quad n_{A}=\frac{1}{2} n(1-p)
$$

are the concentrations of polycation and polyanion segments, respectively.

Based on the chemical potential balance, $\mu_{C}=\mu^{*}$, eqs $81 \mathrm{a}$ and 13 we arrive at the following equations defining the total concentration in the adsorbed layer, $n$, and the composition parameter $p$ :

$$
\begin{gathered}
\frac{1}{N} \ln \frac{n(1+p)}{2 n_{b}}-\frac{1}{2}(B-v) n+C n^{2}+\frac{p}{2}\left(B_{e s}-\tilde{v}\right) n=0 \\
n=\frac{3}{8 C}\left[B-v-p^{2}\left(B_{e s}-\tilde{v}\right)\right]
\end{gathered}
$$

For $N \gg 1$ the ideal-gas log-term in eq $83 \mathrm{a}$ can be neglected, and so we get: $p=p^{*}=\frac{2}{3}(1-\sqrt{1-3 A / 4})$ (note that this result for $p^{*}$ coincides with eq 15). Polymer concentration inside the layer is defined by eq $80 \mathrm{~b}$ with $p=p^{*}$ :

$$
n=n^{*}=\frac{3 \Delta}{8 C}\left(A-p^{* 2}\right)=\frac{\Delta}{2 C}\left(A-p^{*}\right)
$$

The chemical potential of PA segments is (cf. eq 81b) $\mu_{A}^{*} \simeq-\operatorname{Tn}^{*} p^{*} \Delta$, so the potential barrier holding the PA chains in the layer is $U_{A}^{*} \simeq T N n^{*} p^{*} \Delta$. Therefore, their desorption can be neglected if

$$
U_{A}^{*} / T \simeq N n^{*} p^{*}\left(B_{e s}-\tilde{v}\right) \gg 1
$$


which is true for sufficiently long chains, $N \gg\left[n^{*} p^{*}\left(B_{e s}-\tilde{v}\right)\right]^{-1}$.

At the next deposition step of the layer-by-layer adsorption the bulk should contain polyanions, so the picture becomes mirrored: the concentrations of polycations and polyanions after this step are defined by eq 82 with $p=-p^{*}$. The new layer thickness, $L_{k+1}$, is now defined by the condition that the total amount of polycations in the layer does not change, while their concentration changes from $n_{C}=\frac{1}{2} n\left(1+p^{*}\right)$ to $n_{C}=\frac{1}{2} n\left(1-p^{*}\right)$. These conditions lead to

$$
\frac{1}{2} n\left(1-p^{*}\right) L_{k+1}=\frac{1}{2} n\left(1+p^{*}\right) L_{k} \quad \text { or } \quad L_{k+1}=\frac{1+p^{*}}{1-p^{*}} L_{k}
$$

Therefrom we arrive at the exponential growth of the layer thickness

$$
L_{k}=L_{0} e^{k \ln \left(\frac{1+p^{*}}{1-p^{*}}\right)}
$$

Here $L_{0}$ is the initial layer thickness. Note that Eq. (86a) agrees with the empirical eq 1 of ref. 45 .

\section{Discussion and Conclusions}

\section{Discussion}

In the present paper we aimed at considering the most general and universal features of phase equilibria and other phenomena (like layer-by-layer adsorption) in solutions of oppositely charged PEs. For this reason we focused on the structural properties whose characteristic lengthscales (the surface and interfacial layer thickness $\Delta_{\mathrm{i}}$, cf. eq. $45 \mathrm{~b}$, the Debye screening length $r_{D}$, 
the period $\Lambda$ of microdomain structure) which are much longer than the microscopic lengthscales related to small ion or monomer sizes (the Bjerrum length $l_{B}$, the chain thickness $d$, its statistical segment $a_{s}$ and Kuhn segment $l_{K}$ ), but are much shorter that the polymer coil size $R_{\text {coil }} \sim a_{s} \sqrt{N}$. These conditions imply the asymptotic regime of long $(N \gg 1)$ and flexible chains, low polymer volume fraction, $\phi_{p} \ll 1$, weak excluded-volume interactions of PE segments and weakly-charged chains (low fraction of charged monomer units, $f \ll 1$ ). The validity of the third virial approximation for interactions of polymer segments (adopted in our study) is also justified by the above conditions. They also ensure that the polymer translational entropy has a negligible effect for the transitions between different homogeneous and microdomain structures (cf. Figs. 1 and 2): the translational free energy per monomer unit is $\sim T / N$, while the relevant interactional energy is $\sim \tilde{v} n T$ (cf. eq. (12)), hence the latter contribution dominates if $\tilde{v} n N \gg 1$ which is equivalent to $R_{c o i l} \gg \Delta_{\mathrm{i}}$. In this regime the effect of the translational entropy boils down to just a minor renormalization of the interaction parameter $\tilde{v}$.

In addition, we assumed that PA and PC chains are geometrically similar (same chain lengths, monomer sizes, and virial coefficients $B, C$ ). While it is not difficult to lift these assumptions, we preferred to minimize the number of essential parameters involved in the theory.

It is worth noting that we used a mean-field theory neglecting fluctuation corrections whose smallness is guaranteed by eqs. (23), (24) demanding a low third virial interaction constant $C$ and low charge degree $f$; these requirements are in harmony with the general conditions described above. Our theory is based on the Edwards-Lifshitz approach ${ }^{63,64}$ to obtain the polymer conformation related part of the free energy (cf. the first term in the r.h.s. of eq. 
(10)). This approach (also known as the self-consistent field theory, SCFT) naturally leads to the well-known Edwards equations for the polymer partition functions. ${ }^{7,65}$ This method is more general that the RPA theory ${ }^{6}$ valid only for weakly-inhomogeneous polymer structures. The RPA free energy is hinged on the $\mathbf{q}$-dependent polymer chain formfactors $\left(S_{F}(\mathbf{q})\right)$ typically defined in terms of the Debye function (of the wave-vector $\mathbf{q}$ ). ${ }^{6}$ Importantly, the effect of polymer formfactors is automatically taken into account by the Edwards-Lifshitz approach. ${ }^{7}$ This can be easily seen for the regime we focus on, which involves the structural length-scales much longer than the Kuhn segment length $\left(l_{K}\right)$ of polymer backbones. It is well known ${ }^{6,65}$ that in this regime the ideal chain formfactor depends on one "flexibility parameter" only, namely, the statistical segment length $a_{s}$. In the case of long polymers considered here the formfactor becomes $S_{F}(\mathbf{q}) \propto 1 /\left(\mathbf{q}^{2} a_{s}^{2}\right),{ }^{6}$ so the corresponding conformational free energy contribution, which is proportional to $1 / S_{F}(\mathbf{q})$, is consistent with the SCFT expression, eq. (3). It is therefore not surprising that our result, eq. (34), for the spinodal line between homogeneous and lamellar coacervate phases agrees with the RPA-based eq. 39 of ref. 43. It is worth emphasizing that the electrostatic screening effects coupled with polymer formfactors are also automatically accounted for by our approach leading, in particular, to a correct prediction of the Lifshitz point location (cf. Fig. 2) in agreement with earlier results. ${ }^{14,15,41,43}$

Using the SCFT approach allows us to describe not only homogeneous and weaklysegregated coacervate phases, but also the more general intermediate and strong segregation regimes. Here we would like to emphasize that the applicability of our theory in these regimes is not compromised by the fact that the well-known Debye-Hückel (DH) approximation was adopted for small ions (its validity is hinged on conditions $r_{D} \gg l_{B}$ and $f \ll c_{s} / n$, where $c_{s} / n$ is the ratio of small ion to polymer volumes). A strong segregation means that the electrostatic potential energy of a PE segment comparable in size with the lamellar period $\Lambda$ is much larger 
than the thermal energy $T=k_{B} T_{a b s}$. This condition is certainly compatible with the DH requirement that the electrostatic energy of a small ion is $\ll k_{B} T_{a b s}$ since the $\Lambda$-segment contains many charged groups $\left(f \Lambda^{2} / a^{2} \gg 1\right.$ as follows from $\phi_{p} \ll 1$ and the conditions of strong segregation, eq. (59)). It is noteworthy that conditions of that sort are ubiquitous in polymer physics: for example, the well-known Flory-Huggins theory ${ }^{6}$ predicts the onset of phase separation in a symmetric polymer mixture at $\chi N=2$ and a strong segregation of polymers at $\chi N \gg 1$ (with energy gain per chain $\gg k_{B} T_{a b s}$ ) whereas the interaction energy gain per monomer stays always low since typically $\chi \ll 1$.

While the present theory concerns macroscopic coacervate phases, it is also applicable to large finite aggregates (polyelectrolyte complexes) involving many PE chains since internal structure of such complexes is similar to that of macro-coacervates (just like equilibrium internal structure of a large globule reflects that of a macroscopic precipitate). ${ }^{7}$

\section{Conclusions.}

1. Considering dilute solutions of both polycation (PC) and polyanion (PA) chains, we developed a simple equilibrium theory of PE coacervation based on a mean-field approach and virial expansion. We studied weakly charged PE chains with small fraction $f$ of charged units, $f \ll 1$, and very large polymerization index, $N \gg 1$. An added low-molecular monovalent salt of concentration $c_{s}$ provides partial screening of electrostatic interactions characterized by the

Debye length $r_{D}$. The other main parameters are: the second virial coefficient $-B \sim b^{3}\left(1-\frac{\Theta}{T}\right)$ characterizing effective attraction of similar neutral segments below $\Theta$-temperature, the virial 
coefficient $\tilde{v}$ reflecting incompatibility of neutral polycation (PC) and polyanions (PA) (penalty for $\mathrm{AC}$ contacts), and $B_{e s}=f^{2} / c_{s}$, the electrostatic contribution to the effective excluded volume for interaction of similar segments which is a measure of electrostatic interactions between polymer segments. The condition $B>0$ means that the solvent is poor for both polymers (PA and $\mathrm{PC}$ ) in the neutral state when $f=0$. Minimization of the free energy allowed us to find the thermodynamic properties and composition of stable asymmetric uniform polymer phases emerging in the solution and to built the corresponding diagrams of state. We analyzed formation of the lamellar structures in the symmetric coacervate phases considering the general case including weak, intermediate and strong segregation regimes. The stability region of the lamellar phase has been identified and shown on the phase diagram. We also developed a simple theory of the exponential growth for polyelectrolyte multilayers obtained with a layer-by-layer (LbL) technique. ${ }^{66}$

2. Considering uniform macroscopic phases we established that polymer coacervation or precipitation from solution is possible if $2 B>\min \left(\tilde{v}, B_{e s}\right)$. In this regime, the PEs condense and separate into two macroscopic polymer phases (nearly pure PC and PA) if $\tilde{v}>B_{e s}$ and $2 B>B_{e s}$ (cf. region II in Fig. 1a,b). Otherwise, a coacervate phase is emerged. Its nature crucially depends on the dimensionless parameter $A=\frac{2 B-\tilde{v}}{B_{e s}-\tilde{v}}$, where $0<A<\infty$, and on the gross composition of the system $p=\frac{\mathcal{N}_{C}-\mathcal{N}_{A}}{\mathcal{N}_{C}+\mathcal{N}_{A}}$, where $\mathcal{N}_{C}, \mathcal{N}_{A}$ are the total amounts of PC and PA segments, respectively. In particular, for $0<A<1$ (region III in Fig. 1a) the PEs form a single symmetric (if $p=0$ ) or asymmetric (if $0<|p|<p^{*}$, cf. eq 15) macroscopic coacervate phase (note, $p^{*}=p^{*}(A)$ increases with increasing $A$ up to the value $p^{*}=1 / 3$ at $\left.A=1\right)$. For $|p|>p^{*}$ the coacervate phase stays at the critical composition equals to $\pm p^{*}$, while an excess amount of PC 
(if $p>p^{*}$ ) or PA (if $p<-p^{*}$ ) remains in the dilute solution phase. In the region $1<A<3$ (region IV in Fig. 1a) a single coacervate phase is formed for $|p|<p_{c}$ (cf. eq 16), while for $|p|>p_{c}$ the PEs form 2 macrophases: asymmetric coacervate with composition parameter $\pm p_{c}$ and a pure PE phase (PC or PA). A single coacervate phase is always formed for $3<A<\infty$ (region V). Remarkably, the parameter $A$ increases on adding salt, so a transition from region III to region II (in the symmetric case) or a series of transitions III $\rightarrow$ IV $\rightarrow$ V $\rightarrow$ II for $0<A<1$, $\mathrm{IV} \rightarrow \mathrm{V} \rightarrow \mathrm{II}$ for $1<A<3$ (in the composition-asymmetric systems) are expected as the ionic strength of the solution is increased. It means that with increasing salinity the coacervate phase should ultimately separate into pure PC and pure PA phases.

3. Further, we established that the assumption of uniform coacervate phases is valid in the highsalt regime $r_{D}<\Delta_{e s}$, where $\Delta_{e s}=a / \sqrt{n B_{e s}}$ is the characteristic thickness of an interface between PA and PC solutions with $\tilde{v}=B_{e s}$. In the opposite case, $X \equiv r_{D} / \Delta_{e s}>1$, a coacervate with microdomain structure may be thermodynamically more favorable. Focusing on the lamellar morphology we found that for the symmetric case, $p=0$, a lamellar phase is stable if $r *(X)>\frac{\tilde{v}}{B_{e s}}>\frac{2 X-1}{X^{2}}$ with $r *(X) \approx 1+(X-1)^{2} / 4$. Two condensed polyelectrolyte phases (PA and PC) are coexisting at $r^{*}(X)<\tilde{v} / B_{e s}$. The lamellar structure changes from weak to strong as either $\tilde{v} / B_{e s}$ or $X$ is increased. It is interesting to note that the period of the lamellar structure in the case of strong incompatibility between PC and PA chains scales as $\Lambda \sim a \tilde{v}^{1 / 6} f^{-2 / 3}$ and does not depend on the salt concentration. This result is consistent with the corresponding result obtained for a salt-free blend of PC and PA, and the 1/6 power in the dependence of the period on the incompatibility parameter $\tilde{v}$ has been confirmed using DPD simulations. ${ }^{23}$ 
4. As another important result, it is shown that the interfacial tension $\gamma_{\text {inf }}$ between PA and PC phases can become negative at sufficiently low immiscibility parameter $\tilde{v}$. We thus support a similar idea proposed and discussed in ref. 21. We provide an evidence that (both near the Lifshitz point, at $X \approx 1$, and for $X \gg 1$ corresponding to low ion strength, $\left.c_{s} \ll c_{s}^{*}\right)$ the transition from lamellar structure to uniform macroscopic phases occurs at the line of vanishing interfacial tension $\gamma_{\text {inf }}$ (cf. section 6). Exactly at this transition line $\left(\tilde{v} / B_{e s}=r^{*}(X)\right.$, cf. Fig. 2) the macroscopic phases remain stable. Unlike the classical critical phenomena (for example, a liquid/gas coexistence near the critical temperature) the interfacial width remains finite in the present case at the transition line, $\gamma_{\text {inf }}=0$ (cf. eq. (80b)), while the coexisting PA and PC phases stay really distinct. These predictions are likely important: it appears that PE macro-phases with zero (or very small) $\gamma_{\text {inf }}$ must show interesting (anomalous) dynamical behavior.

5. Finally we developed a simple theory of the exponential growth for polyelectrolyte multilayers. We considered the regime (cf. triangular region in Figure 1b) where a nearly uniform macroscopic coacervate phase is thermodynamically stable. This regime is defined by conditions on virial coefficients: $0<\tilde{v}<2 B, B_{e s}>2 B$. In addition, the condition of sufficiently high ionic strength of the solution, $c_{s}>c_{s}^{*}$, must be applied (it is equivalent to $r_{D}<\Delta_{e s}=\frac{a}{\sqrt{n B_{e s}}}$ ), so the lamellar structure does not form. The theory predicts that the PE layer thickness $L_{k}$ increases as a geometrical progression with the number $k$ of deposition steps:

$$
L_{k+1} \simeq K L_{k}
$$


with factor $K=\frac{1+p^{*}}{1-p^{*}}$ and $p^{*}=\frac{2-\sqrt{4-3 A}}{3}$. In the regime we consider $0<A<1,0<p^{*}<1 / 3$ and $1<K<2$. The dependence of $K$ on the ionic strength $c_{s}$ comes from $B_{e s}$ : a higher $c_{s}$ leads to larger $K$ and, therefore, to a more rapid exponential growth of the layer thickness and mass. We thus predict that the regime of exponential growth turns on beyond a critical ionic strength, $c_{s}>c_{s}^{*}$, and that the growth gets faster with an increase of $c_{s}$. Moreover, $K$ also increases, as the polymer charge density $f$ gets lower. These predictions are in harmony with numerous experimental data on PE multilayers. ${ }^{3,45,48,67-71}$

It is worth noting that in the above analysis we assumed that during the deposition time the PE chains penetrate inside the whole multilayer. Obviously, when the film thickness becomes large enough, the molecules will only partially penetrate and the amount of the adsorbed mass becomes dependent on the deposition time. Therefore the growth of the film thickness should eventually follow a linear law.

\section{Notes}

The authors declare no competing financial interest.

\section{ACKNOWLEDGEMENTS}

A.V.S. acknowledges financial support from the Russian Science Foundation (Grant \# 20-1900194). A.N.S. acknowledges a partial support from the International Research Training Group (IRTG) "Soft Matter Science: Concepts for the Design of Functional Materials". 


\section{References:}

1. Richardson, J. J.; Cui, J.; Björnmalm, M.; Braunger, J. A.; Ejima, H.; Caruso, F. Innovation in Layer-by-Layer Assembly. Chem. Rev. 2016, 116, 14828-14867.

2. Blocher, W. C.; Perry, S. L. Complex Coacervate-based Materials for Biomedicine. WIREs Nanomedicine and Nanobiotechnology 2017, 9, 1-28.

3. Das, B. P.; Tsianou, M. From polyelectrolyte complexes to polyelectrolyte multilayers: Electrostatic assembly, nanostructure, dynamics, and functional properties. Adv. Coll. Interface Sci. 2017, 244, 71-89.

4. Izumrudov, V. A.; Mussabayeva, B. Kh.; Murzagulova, K. B. Polyelectrolyte multilayers: preparation and applications. Russ. Chem. Rev., 2018, 87, 192-200.

5. Muthukumar, M. 50th Anniversary Perspective: A Perspective on Polyelectrolyte Solutions. Macromolecules 2017, 50, 9528-9560.

6. de Gennes P.G. Scaling concepts in polymer physics; Cornell University Press: Ithaca, NY,1979.

7. Grosberg, A. Yu.; Khokhlov, A. R. Statistical Physics of Macromolecules; AIP Press: New York, 1994.

8. Khokhlov, A.R.; Khachaturian, K.A. On the theory of weakly charged polyelectrolytes. Polymer 1982, 23, 1742-1750.

9. Dobrynin, A.V.; Rubinstein, M.; Obukhov, S.P. Cascade of transitions of polyelectrolytes in poor solvents. Macromolecules 1996, 29, 2974-2979.

10. Lyulin, A.V.; Dünweg, B.; Borisov, O.V.; Darinskii, A.A. Computer simulation studies of a single polyelectrolyte chain in poor solvent. Macromolecules 1999, 32, 3264-3278.

11. Dobrynin, A.V.; Rubinstein, M. Theory of polyelectrolytes in solutions and at surfaces. Progress in Polymer Science 2005, 30, 1049-1118.

12. Liao, Q.; Dobrynin, A.V.; Rubinstein, M. Counterion-correlation-induced attraction and necklace formation in polyelectrolyte solutions: Theory and simulations. Macromolecules 2006, 39, 1920-1938.

13. Odijk, T. Polyelectrolytes near the rod limit. J. Polym. Sci. Part B: Polym. Phys. 1977, $15,477-483$.

14. Boryu, V. Yu.; Erukhimovich, I. Ya. Structural phase transitions in weakly charged polyelectrolyte solutions. Dokl. Akad. Nauk SSSR. 1986, 286, 1373-1376. 
15. Borue, V. Y.; Erukhimovich, I. Ya. A statistical theory of weakly charged polyelectrolytes: fluctuations, equation of state and microphase separation. Macromolecules 1988, 21, 3240-3249.

16. Joanny, J-F; Leibler L. Weakly charged polyelectrolytes in a poor solvent. J Phys. 1990, $51,545-557$.

17. Khokhlov, A.R.; Nyrkova, I.A. Compatibility enhancement and microdomain structuring in weakly charged polyelectrolyte mixtures. Macromolecules 1992, 25, 1493-1502.

18. Dormidontova, E.E.; Erukhimovich, I.Ya.; Khokhlov, A.R. Microphase separation in poor-solvent polyelectrolyte solutions: Phase diagram. Macromol. Theory and Simul. 1994, 3, 661-675.

19. Rumyantsev A.M.; Kramarenko E.Yu. Two Regions of Microphase Separation in IonContaining Polymer Solutions. Soft Matter. 2017, 13, 6831-6844.

20. Dobrynin, A.V.; Erukhimovich, I.Y. Weak crystallization and structural phase transitions in weakly charged polyelectrolyte systems. Soviet physics, JETP 1991, 72, 751-759.

21. Nyrkova, I.A.; Khokhlov, A.R.; Doi M. Microdomain structures in polyelectrolyte systems: calculation of the phase diagrams by direct minimization of the free energy. Macromolecules 1994, 27, 4220-4230.

22. Rumyantsev, A. M.; de Pablo J. J. Microphase Separation in Polyelectrolyte Blends: Weak Segregation Theory and Relation to Nuclear "Pasta". Macromolecules 2020, 53, 12811292.

23. Rumyantsev, A. M.; Gavrilov, A. A.; Kramarenko, E.Yu. Electrostatically Stabilized Microphase Separation in Blends of Oppositely Charged Polyelectrolytes. Macromolecules 2019, 52, 7167-7174.

24. Borisov, O.V.; Zhulina, E.B. Morphology of micelles formed by diblock copolymer with a polyelectrolyte block. Macromolecules 2003, 36, 10029-10036.

25. Nyrkova, I. A.; Semenov, A. N. On the theory of aggregation and micellization:PEOPVP copolymer in water. Faraday Discuss. 2005, 128, 113-127.

26. Dobrynin, A.V.; Colby, R.H.; Rubinstein, M. Polyampholytes. J. Polym. Sci. Part B: Polym. Phys. 2004, 42, 3513-3538.

27. Gucht, J. van der; Spruijt, E.; Lemmers, M.; Cohen Stuart, M. A. Polyelectrolyte complexes: Bulk phases and colloidal systems. J. Colloid Interface Sci. 2011, 361, 407-422. 
28. Li, L.; Srivastava, S.; Andreev, M.; Marciel, A. B.; de Pablo, J. J.; Tirrell, M. V. Phase Behavior and Salt Partitioning in Polyelectrolyte Complex Coacervates. Macromolecules 2018, 51, 2988-2995.

29. Sing, C. E.; Perry, S. L. Recent Progress in the Science of Complex Coacervation. Soft Matter 2020, 16, 2885-2914.

30. Overbeek, J. T. G.; Voorn, M. J. Phase separation in polyelectrolyte solutions. Theory of complex coacervation. J. Cell. Comp. Physiol. 1957, 49, 7-26.

31. Michaeli, I.; Overbeek, J. T. G.; Voorn, M. J. Phase separation of polyelectrolyte solutions., J. Polym. Sci. 1957, 23, 443-450.

32. Borue, V. Y.; Erukhimovich, I. Ya. A statistical theory of globular polyelectrolyte complexes. Macromolecules 1990, 23, 3625-3632.

33. Castelnovo, B. M.; Joanny, J. F. Phase Diagram of Diblock Polyampholyte Solutions. Macromolecules 2002, 35, 4531-4538.

34. Kudlay, A.; Olvera de la Cruz, M. Precipitation of oppositely charged polyelectrolytes in salt solutions. J. Chem. Phys. 2004, 120, 404-412.

35. Rumyantsev, A.M.; Potemkin I.I. Explicit Description of Complexation between Oppositely Charged Polyelectrolytes as an Advantage of the Random Phase Approximation Over the Scaling Approach. Phys. Chem. Chem. Phys. 2017, 19, 27580-27592.

36. Shusharina, N.P.; Zhulina, E.B.; Dobrynin, A.V.; Rubinstein, M. Scaling theory of diblock polyampholyte solutions. Macromolecules 2005, 38, 8870-8881.

37. Rubinstein, M.; Liao, Q.; Panyukov, S. Structure of liquid coacervates formed by oppositely charged polyelectrolytes. Macromolecules 2018, 51, 9572-9588.

38. Delaney, K. T.; Fredrickson, G. H. Theory of polyelectrolyte complexation - Complex coacervates are self-coacervates. J. Chem. Phys. 2017, 146, 224902.

39. Danielsen, S.P.O.; McCarty, J.; Shea, J.E.; Delaney, K.T.; Fredrickson, G.H. Small ion effects on self-coacervation phenomena in block polyampholytes. J. Chem. Phys. 2019, 151, 034904.

40. Zhang, P; Alsaifi, N. M.; Wu, J.; Wang, Z-J. Polyelectrolyte complex coacervation: Effects of concentration asymmetry. J. Chem. Phys. 2018, 149, 163303.

41. Castelnovo, B. M.; Joanny, J. F. Complexation between oppositely charged polyelectrolytes: Beyond the Random Phase Approximation. Eur. Phys. J. E 2001, 6, 377-386.

42. Rumyantsev, A.M.; Zhulina, E.B.; Borisov, O.V. Complex Coacervate of Weakly Charged Polyelectrolytes: Diagram of States. Macromolecules 2018, 51, 3788-3801. 
43. Rumyantsev, A.M.; Kramarenko, E.Yu.; Borisov, O.V. Microphase Separation in Complex Coacervate Due to Incompatibility between Polyanion and Polycation. Macromolecules. 2018, 51, 6587-6601.

44. Schlenoff, J. B.; Dubas, S. T. Mechanism of Polyelectrolyte Multilayer Growth: Charge Overcompensation and Distribution. Macromolecules 2001, 34, 592-598.

45. Picart, C.; Mutterer, J.; Richert, L.; Luo, Y.; Prestwich, G. D.; Schaaf, P.; Voegel, J.-C.; Lavalle, P. Molecular basis for the explanation of the exponential growth of polyelectrolyte multilayers. PANS 2002, 99, 12531-12535.

46. Schonhoff, M.; Self-assembled polyelectrolyte multilayers. Curr. Opin. Colloid Interface Sci. 2003, 8, 86-95.

47. Klitzing, R. V. Internal structure of polyelectrolyte multilayer assemblies. Phys. Chem. Chem. Phys. 2006, 8, 5012-5033.

48. Salehi, A.; Desai, P. S.; Li, J.; Steele, C. A.; Larson, R. G. Relationship between Polyelectrolyte Bulk Complexation and Kinetics of Their Layer-by-Layer Assembly. Macromolecules 2015, 48, 400-409.

49. Boulmedais, F.; Ball, V.; Schwinte, P., Frisch, B.; Schaaf, P.; Voegel, J.-C. Buildup of Exponentially Growing Multilayer Polypeptide Films with Internal Secondary Structure. Langmuir 2003, 19, 440-445.

50. Laugel, N.; Betscha, C.; Winterhalter, M.; Voegel, J.-C.; Schaaf, P.; Ball, V. Relationship between the growth regime of polyelectrolyte multilayers and the polyanion/polycation complexation enthalpy. J.Phys.Chem. B 2006, 110, 19443.

51. O. I. Vinogradova, O.V. Lebedeva, K. Vasilev, H. Gong, J. Garcia-Turiel, and B.-S. Kim, Multilayer DNA/Poly(allylamine hydrochloride) Microcapsules: Assembly and Mechanical Properties. Biomacromolecules 2005, 6, 1495-1502.

52. Porcel, C.; Lavalle, P.; Ball, V.; Decher, G.; Senger, B.; Voegel, J.-C.; Schaaf, P. From Exponential to Linear Growth in Polyelectrolyte Multilayers. Langmuir 2006, 22, 4376-4383.

53. Netz R. R.; Andelman D.; Neutral and charged polymers at interfaces. Phys. Rep. 2003, $380,1-95$.

54. Grosberg, A. Yu.; Nguyen, T. T.; Shklovskii, B. I. Colloquium: The physics of charge inversion in chemical and biological systems. Rev. Mod. Phys. 2002, 74, 329-345.

55. Joanny, J.-F. Polyelectrolyte adsorption and charge inversion. Eur. Phys. J. B 1999, 9, 117-122. 
56. Dobrynin, A. V.; Rubinstein, M. Effect of short-range interactions on polyelectrolyte adsorption at charged surfaces. J. Phys. Chem. B 2003, 107, 8260-8269.

57. Winkler, R. G.; Cherstvy, A. G. Strong and Weak Polyelectrolyte Adsorption onto Oppositely Charged Curved Surfaces. Adv. Polym. Sci. 2014, 255, 1-56.

58. Subbotin A.V.; Semenov A.N. Adsorption of flexible polyelectrolytes on charged surfaces. Soft Matter 2016, 12, 6771-6787.

59. Castelnovo, M.; Joanny, J.-F. Formation of Polyelectrolyte Multilayers. Langmuir 2000, $16,7524-7532$.

60. Patel, P.A.; Jeon, J.; Mather, P.T.; Dobrynin, A.V. Molecular dynamics simulations of multilayer polyelectrolyte films: effect of electrostatic and short-range interactions. Langmuir 2006, 22, 9994-10002.

61. Shafira, A.; Andelman, D. Polyelectrolyte multilayer formation: Electrostatics and shortrange interactions. Eur. Phys. J. E 2006, 19, 155-162.

62. Landau, L.D.; Lifshitz, E.M. Course of Theoretical Physics, Statistical Physics, Part 1; Pergamon Press, New York,1980.

63. Edwards, S.F. The theory of polymer solutions at intermediate concentration. Proc.Phys. Soc. 1966, 88, 265-280.

64. Lifshitz, I.M. Some Problems of the Statistical Theory of Biopolymers. Sov.Phys. JETP, 1969, $28,1280-1286$.

65. Fredrickson G. H. The Equilibrium Theory of Inhomogeneous Polymers; Clarendon Press, Oxford, 2006.

66. Decher, G.; Eckle, M.; Schmitt, J.; Struth, B. Layer-by-layer assembled multicomposite films. Current Opinion Colloid Interface Sci. 1998, 3, 32-39.

67. Tang K.; Besseling, N.A.M. Formation of polyelectrolyte multilayers: ionic strengths and growth regimes. Soft Matter 2016, 12, 1032-1040.

68. Ruths, J.; Essler, F.; Decher, G.; Riegler, H. Polyelectrolytes I: Polyanion/Polycation Multilayers at the Air/Monolayer/Water Interface as Elements for Quantitative Polymer Adsorption Studies and Preparation of Hetero-superlattices on Solid Surfaces. Langmuir 2000, $16,8871-8878$.

69. McAloney, R. A.; Sinyor, M.; Dudnik V.; Goh, M. C. Atomic Force Microscopy Studies of Salt Effects on Polyelectrolyte Multilayer Film Morphology. Langmuir 2001, 17, 6655-6663. 
70. C. Picart, C. Gergely, B. Senger, Y. Arntz, J. C. Voegel, P. Schaaf and F. J. G. Cuisinier, Measurement of film thickness up to several hundreds of nanometers using optical waveguide lightmode spectroscopy. Biosens. Bioelectron., 2004, 20, 553-561.

71. Richert, L.; Lavalle, P.; Payan, E.; Shu, X. Z.; Prestwich, G. D.; Stoltz, J.-F.; Schaaf, P.; Voegel, J.-C.; Picart, C. Layer by Layer Buildup of Polysaccharide Films: Physical Chemistry and Cellular Adhesion Aspects. Langmuir, 2004, 20, 448-458. 